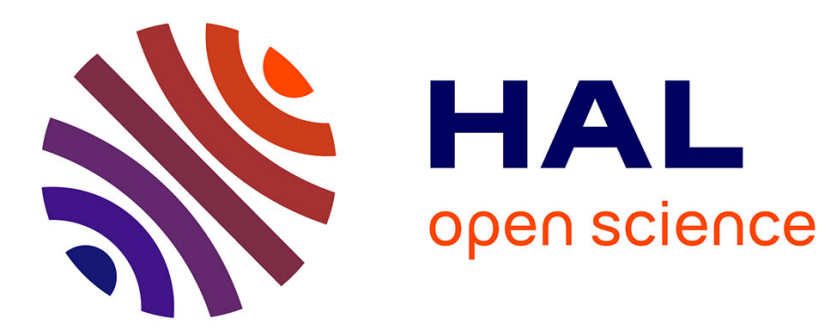

\title{
Contribution to generic modeling and vision-based control of a broad class of fully parallel robots
}

Tej Dallej, Nicolas Andreff, Philippe Martinet

\section{To cite this version:}

Tej Dallej, Nicolas Andreff, Philippe Martinet. Contribution to generic modeling and visionbased control of a broad class of fully parallel robots. Robotica, 2018, 36 (12), pp.1874 - 1896. 10.1017/S0263574718000784 . hal-02399261

\section{HAL Id: hal-02399261 \\ https://hal.science/hal-02399261}

Submitted on 9 Dec 2019

HAL is a multi-disciplinary open access archive for the deposit and dissemination of scientific research documents, whether they are published or not. The documents may come from teaching and research institutions in France or abroad, or from public or private research centers.
L'archive ouverte pluridisciplinaire HAL, est destinée au dépôt et à la diffusion de documents scientifiques de niveau recherche, publiés ou non, émanant des établissements d'enseignement et de recherche français ou étrangers, des laboratoires publics ou privés. 


\section{Contribution to generic modeling and vision-based control of a broad class of fully parallel robots Tej Dallej $\triangleleft^{*}$,Nicolas Andreff $\triangleleft \triangleright$ and Philippe Martinet $\triangleleft \S$}

$\triangleleft$ Institut Pascal, UBP/CNRS/SIGMA, Clermont-Ferrand, France.

$\triangleright$ Institut FEMTO-ST, Univ. Franche-Comté/CNRS/ENSMM/UTBM, Besançon, France.

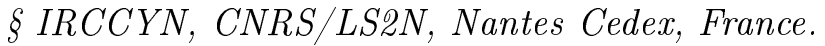

)

\section{SUMMARY}

This paper deals with a generic modeling and vision-based control approach for a broad class of parallel mechanisms. First, a generic architecture representing several families is proposed. Second, inspired by the geometry of lines, a generic differential inverse kinematic model according to the proposed generic structure is introduced. Finally, based on the image projection of cylindrical legs, a kinematic vision-based control using legs observation is presented. The approach is illustrated and validated on the Gough-Stewart and Par4 parallel robots.

KEYWORDS: Visual Servoing; Control; Modeling; Parallel Robots; Kinematics.

\section{Introduction}

The coupling between visual servoing techniques ${ }^{1-3}$ and parallel robots is becoming increasingly important and has been the subject of several studies. Essentially, kinematic vision-based control generates a Cartesian desired velocity, which is converted into joint velocities by the differential inverse kinematic model. The latter is usually an analytical model in the case of parallel robots. The differential inverse kinematic model also depends on the Cartesian pose, ${ }^{4,5}$ which needs to be estimated.

Authors in refs. [6,7] have been among the first to introduce vision-based control for parallel robots. The interesting point of the aforementioned approaches is the use of vision in the feedback control for regulation. ${ }^{8}$ However, the used parallel robots are designed with particular mechanical structures to have an analytical forward kinematic model. Consequently, the differential inverse kinematic model turns out to be dependent on the end-effector pose estimated using the forward kinematic model and joint values.

In actuality, the formulation of the parallel robot forward kinematic model ${ }^{9}$ is generally difficult to solve and gives several possible poses for the end-effector. ${ }^{10}$ To make the control robust with respect to modeling errors, vision replaces the forward kinematic model in the feedback with a camera measurement. This method can also be used to simplify different models of the control scheme. ${ }^{8}$ Amongst the various visual servoing techniques, $3 \mathrm{D}$ pose visual servoing ${ }^{11,12}$ can be applied to parallel robots. In ref. [13], a generic 3D pose visual servoing of six degrees of freedom (DOF) Gough-Stewart platform (Fig. 1) was proposed. In this kinematic control, the end-effector pose, indirectly measured, was used in the feedback control and in the differential inverse kinematic model.

Image Based Visual Servoing (IBVS) was also applied to parallel robots ${ }^{14,15}$ using endeffector observation. This method does not require the model of the visual target or the end-effector pose. However, the method has difficulties with large rotational motion. ${ }^{16}$ The main limitation of visual servoing of parallel robots focused on the observation of a

\footnotetext{
* Corresponding author. E-mail: tdallej@yahoo.fr
} 

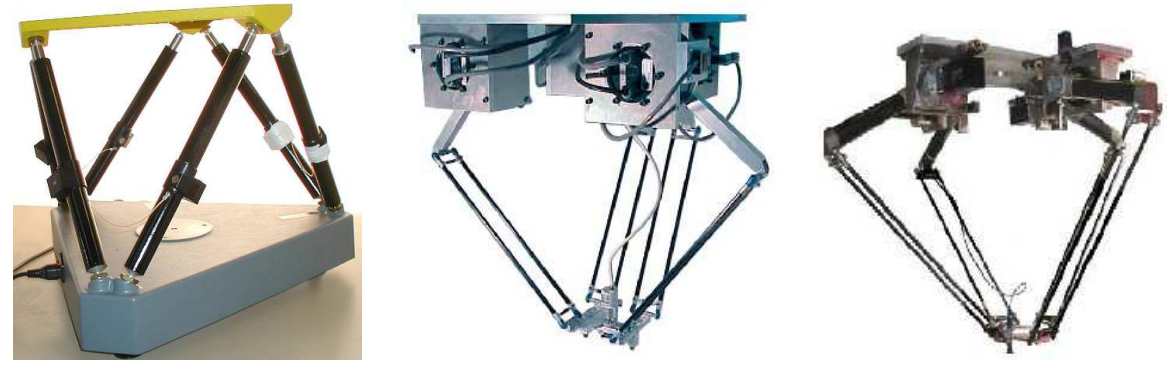

Fig. 1. From left to right: the Gough-Stewart platform (Institut Pascal), the H4 and the Par4 robots (LIRMM).
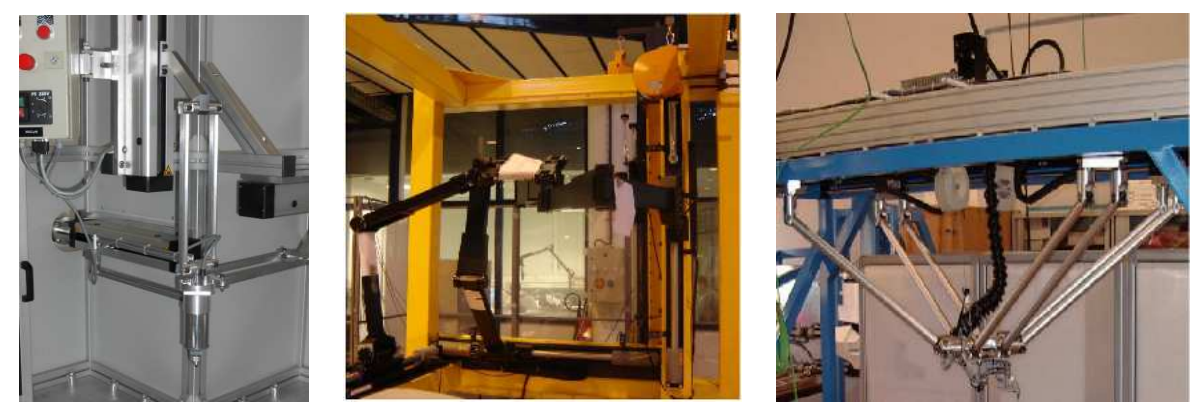

Fig. 2. From left to right: the Orthoglide (IRCCyN), the Isoglide-4 T3R1 (IFMA) and the I4L (LIRMM) robots.

visual target is that it requires the estimation of the end-effector to tool transformation, the world to base transformation and the entire kinematic parameter set. Moreover, it is not wise to consider observing the end-effector of a machining tool. It may be incompatible with various applications.

It is noticeable that the state of a parallel robot is any representation of the end-effector pose. A new way to use vision which gathers the advantages of redundant metrology ${ }^{17,18}$ and visual servoing was presented in ref. [19]. This method was proposed to control the well-known six degrees of freedom (DOF) Gough-Stewart platform using legs observation. This proposed approach has a reduced set of kinematic parameters and does not require any visual target. The orientation of the robot's legs was chosen as visual primitives. The control law was based on their reconstruction from the image which might not be very accurate for intrinsic reasons. To improve the practical robustness by servoing the legs in the image, it was proposed in ref. [20] to servo leg edge rather than leg orientation. The proposed method was extended to Par4 ${ }^{21}$ and I4L ${ }^{22}$ robots (Fig. 1 and Fig. 2). In ref. [23], the authors suggested a dynamic control via leg edges of a parallel robot taking into account all relevant aspects of leg edges observation.

The notion that the legs can contain the state of a parallel robot leads the way towards an innovative approach in which modeling, calibration ${ }^{24}$ and control are interlaced. In addition, it can permit a better representation of a parallel robot. References $[25,26]$, show that observing the direction of the parallel robot's legs involves controlling the displacement of a hidden robot. The latter has up to eight assembly modes that are different from those of the real live robot. First, the method shown in ref. [19] was used for control. Afterwards, based on the kinematic modeling of the hidden robot, the singularity problem of the controller was studied and controllability information of the parallel robot was given.

In our opinion, a generic solution for controlling any parallel robot should take into account the specific kinematic properties of parallel robots and the observable kinematic elements. This solution does not require any additional visual target. The terminology of a kinematic element was first introduced in ref. [27] in order to find geometric and kinematic properties which can be applied to many parallel robots. The indicated representative 
kinematic chain is made up of at least one kinematic element which is composed of two rigid bodies linked by a Prismatic joint (fixed or moving joint).

The notion of a kinematic element is then fully formalized in ref. [28] and an interesting mathematical model of a kinematic element was given. The proposed method gives a linear dynamic modeling of parallel robot from observable kinematic elements. It relies on a body-oriented representation of observable rectilinear kinematic structures. However, the modeling and control of parallel robots on a kinematic level were not clearly and fully studied. In addition, the proposed modeling method in ref. [28] was illustrated on a simple 2 degrees of freedom (DOF) five-bar mechanism which is an RRR-RR structure planar parallel robot. Some discussion about the extension to a broad class of parallel mechanisms would have been more interesting, complete and generic.

Confident that one can fuse kinematics and projective geometry into a projective kinematic model for control and inspired by the geometry of lines and the image projection of cylindrical legs, this paper has three main goals:

- to propose a generic structure of the kinematic chains and the nacelle ${ }^{29-31}$ (an articulated set of elements having fixed length and containing the moving platform). It is a general way to find generic parallel robot structure corresponding to a broad class of fully parallel robots, e.g. the Gough-Stewart Platform, the H4 ${ }^{29}$ the I4R, ${ }^{30}$ the Par4 ${ }^{31}$ (Fig. 1) and the I4L ${ }^{32}$ (Fig. 2).

- to provide a thorough study of modeling and kinematic vision-based control derived according to a generic methodology taking into account the proposed generic parallel robot structure. The edges of the last elements (referent elements) attached to the nacelle are used in the servo loop and in the kinematic visual servoing law. We will confirm that, measuring by vision the referent elements edges, one can easily measure a projective kinematic model for control without proprioceptive sensors, which might simplify the robot design.

- to present a coherent representation of a broad class of fully parallel robots, in which one can fuse a generic architecture, the kinematic modeling and referent elements observation.

The paper is organised as follows: Section 2 presents a comparative study of some existing parallel robots. The aim is to find similar points and to introduce a generic architecture. Based on some assumptions and the structures of different parallel robot families, section 3 presents a generic architecture of a broad class of fully parallel robots with one actuated joint. It is composed of the kinematic chains architectures and the nacelle including the moving platform. Section 4 introduces the concept of the observable referent kinematic elements. The projection of cylindrical elements in the image gives the visual primitives used for the edge-based control. Section 5 explains the generic differential inverse kinematic model according to the proposed generic structure. A kinematic visionbased control using referent elements observation is then presented in section 6 . Section 7 presents experimental validations on the Gough-Stewart platform and the Par4 robot. A discussion and conclusions are presented in sections 8 and 9, respectively.

\section{Structure of some parallel robot families}

As we plan to propose a generic architecture which unifies several families of parallel robots, specific kinematic properties of a broad class of parallel robots must be found. The generic unified method must be able to be used for more than one type of parallel robot to prove the validity of the proposed approaches. Consequently, this section deals with a comparative study of some existing parallel robots. The main objective is to find a common representation and to introduce a generic architecture for a broad class of parallel robots. This classification is based on the presence or absence of a Prismatic joint in each kinematic chain. ${ }^{24,33}$

Throughout the paper, the notations given in Table I will be used. 


\subsection{First family}

The first family includes robots whose actuated Prismatic joint is located between two rigid bodies of the kinematic chain. For example, the Gough-Stewart platform (Fig. 3) is a six degrees of freedom parallel mechanism. It has 6 kinematic chains $\left[\mathbf{A}_{1 i} \mathbf{A}_{2 i}\right]$ with varying lengths $L\left(r_{1 i}\right)=r_{1 i}, i \in 1 . .6$ due to the actuated Prismatic joints located at $\mathbf{P}_{1 i}$. The kinematic chains of a standard Gough-Stewart platform are attached to the base by Universal joints (located at points $\mathbf{A}_{1 i}$ ) and to the moving platform by Ball joints located at points $\mathbf{A}_{2 i}$. The moving platform of the Gough-Stewart robot is made up of a single element $\mathcal{S}$ attached to each kinematic chain at point $\mathbf{A}_{2 i}$.
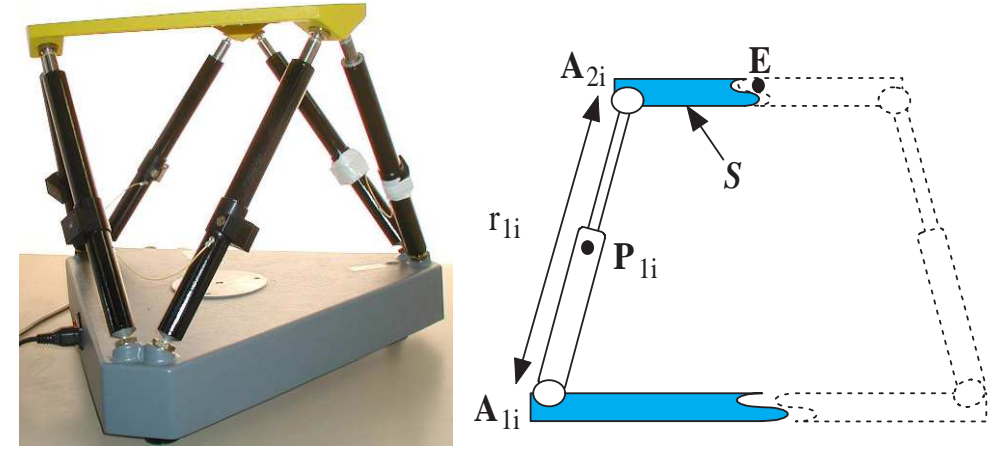

Fig. 3. A real Gough-Stewart platform and its representative Sketch.

Table I . Notations.

- Boldface characters denote vectors or matrices. Unit vectors are underlined.

- ${ }^{i} \mathbf{T}_{j}=\left(\begin{array}{cc}{ }^{i} \mathbf{R}_{j} & { }^{i} \mathbf{t}_{j} \\ \mathbf{0} & \mathbf{1}\end{array}\right)$ is the homogeneous matrix associated to the rigid transformation from frame $\mathcal{F}_{i}$ to frame $\mathcal{F}_{j}$.

- ${ }^{i} \mathbf{v}$ is vector $\mathbf{v}$ expressed in frame $\mathcal{F}_{i}$.

- $\mathbf{K}$ is the matrix of the camera's intrinsic parameters.

- $q_{0 i}$ defines the first actuated joint of kinematic chain $i$.

- $\mathbf{q}$ defines the vector of all actuated joints.

- ${ }^{j} \boldsymbol{\tau}_{i}=\left({ }^{j} \mathbf{V}_{i}{ }^{j} \boldsymbol{\Omega}_{i}\right)^{T}$ is the Cartesian velocity (linear and angular velocities) of $\mathcal{F}_{i}$ expressed in and with respect to $\mathcal{F}_{j}$. It can be minimally represented by ${ }^{i} \mathcal{V}_{j}$ when the components of the Cartesian velocity are less than 6.

- $\mathbf{M}^{+}$is the pseudo-inverse of $\mathbf{M}$.

- $\widehat{\mathbf{M}}$ is the estimation of $\mathbf{M}$ based on measurements.

- $[\mathbf{a}]_{\times}$is the cross-product matrix of vector $\mathbf{a}$.

- An element with constant length of a kinematic chain is equivalent to two rigid bodies linked by a fixed Prismatic joint (Fixed joint).

- A kinematic element is considered as two rigid bodies linked by a Prismatic joint (fixed or moving joint).

- Each kinematic chain can be composed of one or more kinematic elements.

- $i=1$..n denotes the kinematic chains (legs), $m=0 . .1$ denotes the kinematic elements which form the kinematic chain and $j=1 . .2$ denotes the edges of each observable cylindrical kinematic element.

- $\mathcal{F}_{b}=\left(\mathbf{O}, \underline{\mathbf{x}}_{b}, \underline{\mathbf{y}}_{b}, \underline{\mathbf{z}}_{b}\right), \quad \mathcal{F}_{e}=\left(\mathbf{E}, \underline{\mathbf{x}}_{e}, \underline{\mathbf{y}}_{e}, \underline{\mathbf{z}}_{e}\right), \quad \mathcal{F}_{c}=\left(\mathbf{O}_{c}, \underline{\mathbf{x}}_{c}, \underline{\mathbf{y}}_{c}, \underline{\mathbf{z}}_{c}\right) \quad$ and $\quad \mathcal{F}_{p m i}=$ $\left(\mathbf{P}_{m i}, \underline{\mathbf{x}}_{p m i}, \underline{\mathbf{y}}_{p m i}, \underline{\mathbf{z}}_{p m i}\right)$ denote the base, end-effector, camera and $i^{\text {th }}$ kinematic element reference frames, respectively.

- The camera frame is fixed with respect to the base frame.

- A nacelle $\mathrm{e}^{29-31}$ is made of an articulated set of kinematic elements (with a fixed length). Only one of these elements is considered as the moving platform of the robot.

- In some cases, a nacelle can be composed of one element (with a fixed length) which is the moving platform.

- The associated variables for each joint are $r_{k i}$ for Prismatic joint, $\alpha_{k i}$ for Revolute joint, $\left(\alpha_{k i}, \beta_{k i}\right)$ for Universal joint and $\left(\alpha_{k i}, \beta_{k i}, \gamma_{k i}\right)$ for Ball joint, with $k=0 . .2$. 


\subsection{Second family}

The second family includes robots which have one or two kinematic elements with a fixed length and an actuated Prismatic joint between these elements and the base. It includes, for instance, the Orthoglide robot, ${ }^{34}$ the Isoglide- $4 \mathrm{~T} 3 \mathrm{R} 1^{35}$ and the $\mathrm{I} 4 \mathrm{~L}^{32}$ (Fig. 2).

2.2.1. The Orthoglide robot. The Orthoglide is a 3-DOF translational parallel manipulator. It consists of three identical kinematic chains attached to the base at points $\mathbf{P}_{0 i}$ by three actuated and orthogonal Prismatic joints.

Upon analyzing in more detail the architecture of the Orthoglide (Fig. 4), one can consider a first kinematic element $\left[\mathbf{A}_{0 i} \mathbf{A}_{1 i}^{1}\right]$ with variable length $l\left(r_{0 i}\right), i \in 1 . .3$. It is connected to the articulated parallelogram composed of two elements $\left[\mathbf{A}_{1 i}^{1} \mathbf{A}_{2 i}^{1}\right]$ and $\left[\mathbf{A}_{1 i}^{2} \mathbf{A}_{2 i}^{2}\right]$ with a fixed length $L\left(r_{1 i}\right)=L$ (Fig. 4).

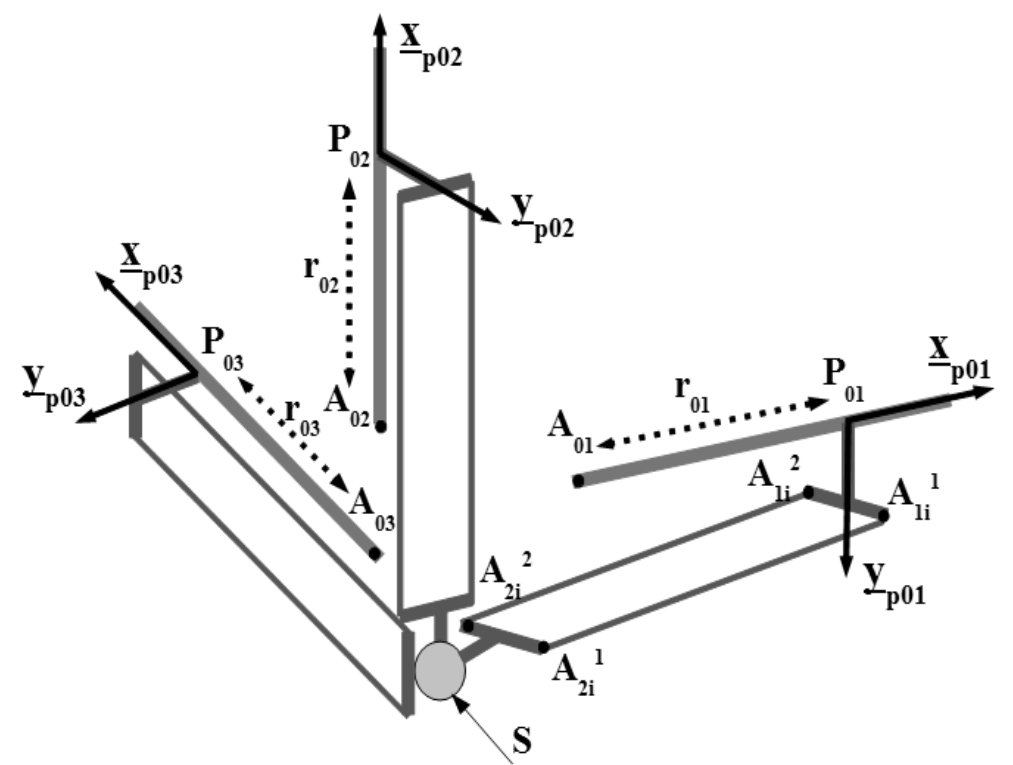

Fig. 4. Sketch of the Orthoglide.

2.2.2. The I4L robot. The I4L robot is composed of 4 actuated Prismatic joints. Each linear motor located at $\mathbf{P}_{0 i}$ moves the kinematic element $\left[\mathbf{P}_{0 i} \mathbf{A}_{1 i}^{1}\right]$. One can consider a first element $\left[\mathbf{A}_{0 i} \mathbf{A}_{1 i}^{1}\right]$ with variable length $l\left(r_{0 i}\right), i \in 1 . .4$. Each kinematic element is connected to an articulated parallelogram (forearm) equipped with ball joints $\left(\mathbf{A}_{1 i}^{1}, \mathbf{A}_{2 i}^{1}\right)$ and $\left(\mathbf{A}_{1 i}^{2}, \mathbf{A}_{2 i}^{2}\right)$ (Fig. 5). The state of the forearm can be defined using points $\mathbf{A}_{1 i}^{1}$ or $\mathbf{A}_{1 i}^{2}$ and $\mathbf{A}_{2 i}^{1}$ or $\mathbf{A}_{2 i}^{2}$, respectively.

The rotation of moving platform $\mathcal{S}$ is due to the relative displacement of the two nacelle parts $\mathcal{S}_{01}=\mathcal{S}_{02}$ and $\mathcal{S}_{03}=\mathcal{S}_{04}$ (Fig. 5), using two rack-and-pinion systems. The relative translation $\mathbf{T}$ is transformed into a proportional end-effector rotation $\theta=\mathbf{T} / \mathcal{K}$, with $\mathcal{K}$ as the transmission ratio. It should be observed that each kinematic chain $i$ is attached to a nacelle element $\mathcal{S}_{0 i}$ which is directly attached to moving platform $\mathcal{S}$ at $\mathbf{D}_{0}$ or $\mathbf{D}_{1}$.

\subsection{Third family}

In the third family, there is no actuated Prismatic joint in the kinematic chains. It includes the H4, the I4R and the Par4 (Fig. 1). All these robots have only actuated Revolute joints. H4, I4R and Par4 robots are based on four identical kinematic chains (Fig. 6, Fig. 7, Fig. 8 and Fig. 9). Each revolute motor located at $\mathbf{A}_{0 i}$ moves arm $i$ with a fixed length $l\left(r_{0 i}\right)=l$, $i \in 1$..4. Each arm is connected to a forearm (parallelogram) made up of two structures equipped with ball joints $\left(\mathbf{A}_{1 i}^{1}, \mathbf{A}_{2 i}^{1}\right)$ and $\left(\mathbf{A}_{1 i}^{2}, \mathbf{A}_{2 i}^{2}\right)$. The axis passing through the two upper ends (or the lower extremities) of this parallelogram keeps the same direction. The state of the forearm can be defined using points $\mathbf{A}_{1 i}^{1}$ or $\mathbf{A}_{1 i}^{2}$ and $\mathbf{A}_{2 i}^{1}$ or $\mathbf{A}_{2 i}^{2}$, respectively. 

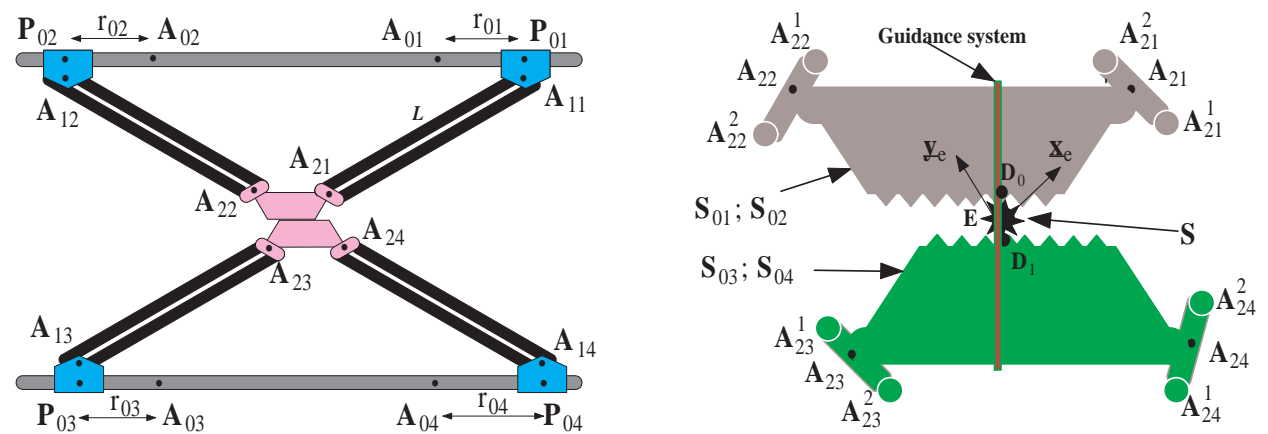

Fig. 5. Top view of the I4L robot (left) and its nacelle (right).

Each forearm is connected at each end to the articulated nacelle. The end-effector frame located at $\mathbf{E}$ can be translated in three directions and rotated around a fixed axis ${ }^{c} \underline{\mathbf{z}}_{e}$.

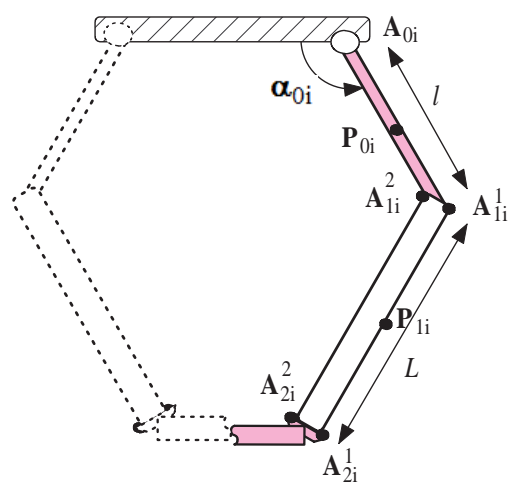

Fig. 6. H4, I4R and Par4 kinematic chain parameters.

2.3.1. The nacelle of the $\mathrm{H}_{4}$ robot. The nacelle of the $\mathrm{H} 4$ (Fig. 7) is composed of two lateral parts and a central rod $\mathcal{S}=\left[\mathbf{D}_{24} \mathbf{D}_{21}\right]$. The center of this rod is the end-effector $\mathbf{E}$ which has 4 DOF (3 translations and 1 rotation).
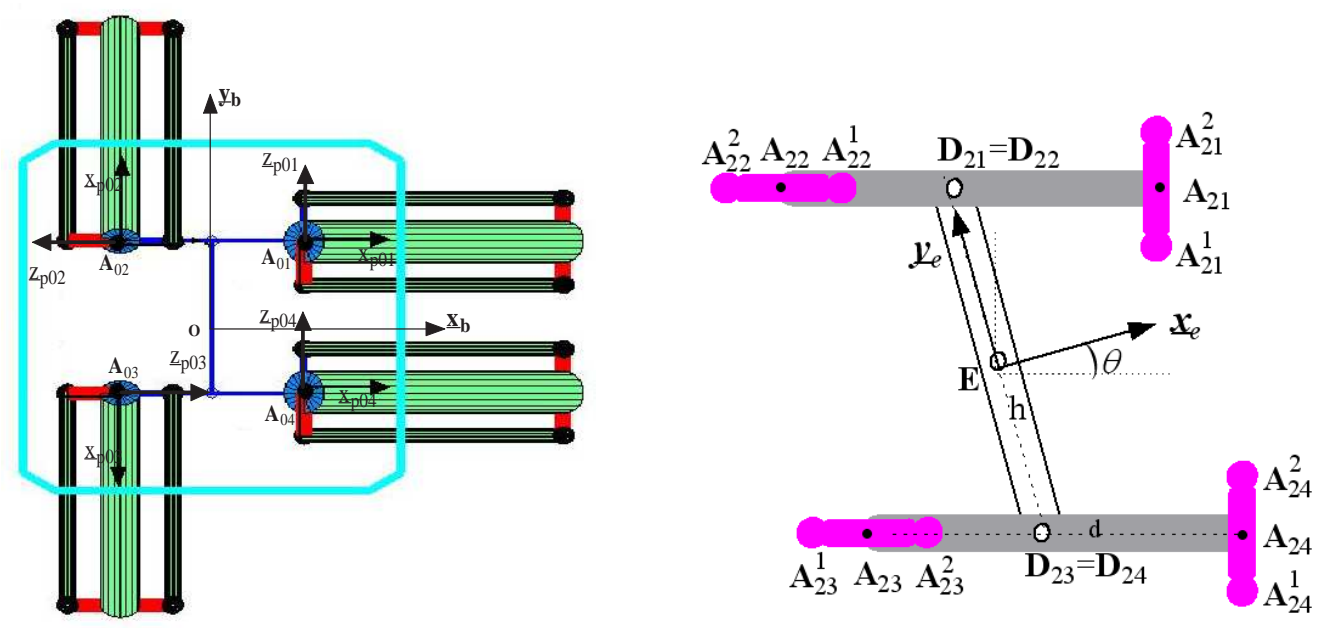

Fig. 7. Top view of the $\mathrm{H} 4$ robot (left) and its nacelle (right).

2.3.2. The nacelle of the $I_{4} R$ robot. The nacelle of I4R robot is made up of 3 articulated elements. The relative displacement of the two plate parts $\mathcal{S}_{01}=\mathcal{S}_{02}$ and $\mathcal{S}_{03}=\mathcal{S}_{04}$ (Fig. 8) 
induces the rotation of the end-effector located at the center of $\mathcal{S}=\left[\mathbf{D}_{0} \mathbf{E}\right]$. The relative translation $\mathbf{T}$ is transformed into a proportional end-effector rotation $\theta=\mathbf{T} / \mathcal{K}$.
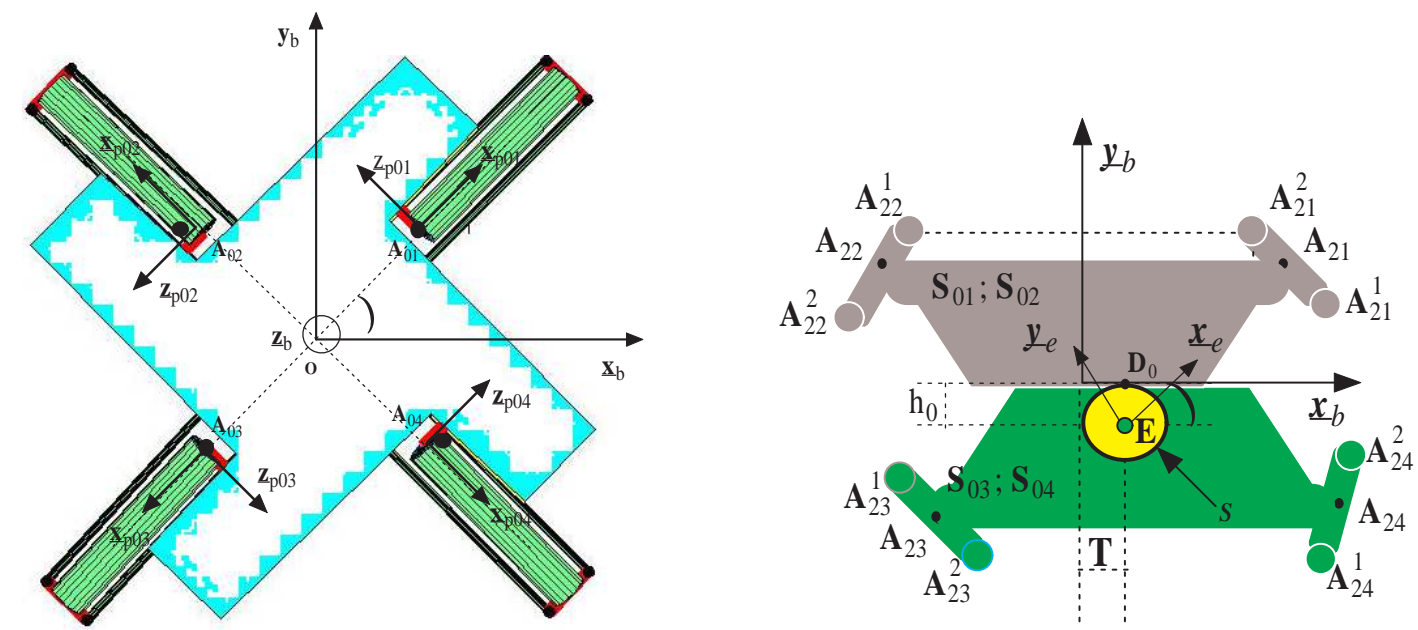

Fig. 8. Top view of the I4R robot (left) and its nacelle (right).

2.3.3. The nacelle of the Par4 robot. As shown in ref. [31], the Par4 nacelle (Fig. 9) is composed of four parts: two parts defined by $\left[\mathbf{D}_{21} \mathbf{D}_{22}\right]$ and $\left[\mathbf{D}_{23} \mathbf{D}_{24}\right]$ and linked by two rods $\left[\mathbf{D}_{21} \mathbf{D}_{24}\right]$ and $\left[\mathbf{D}_{22} \mathbf{D}_{23}\right]$ with revolute joints.

The articulated nacelle is equipped with an amplification system (Fig. 9) to transform the relative rotation $\theta= \pm \frac{\pi}{4}$ of the end-effector at $\mathbf{E}=\mathbf{D}_{24}$ into a proportional rotation $(\beta=-\kappa \theta, \kappa=3)$ of a new end-effector at $\mathbf{E}_{1}$. However, this amplification system is not very significant from a kinematic point of view. Consequently, one can choose $\mathbf{D}_{24}=\mathbf{E}$ as the end-effector position linked to the element $\left[\mathbf{D}_{21} \mathbf{D}_{24}\right]$.
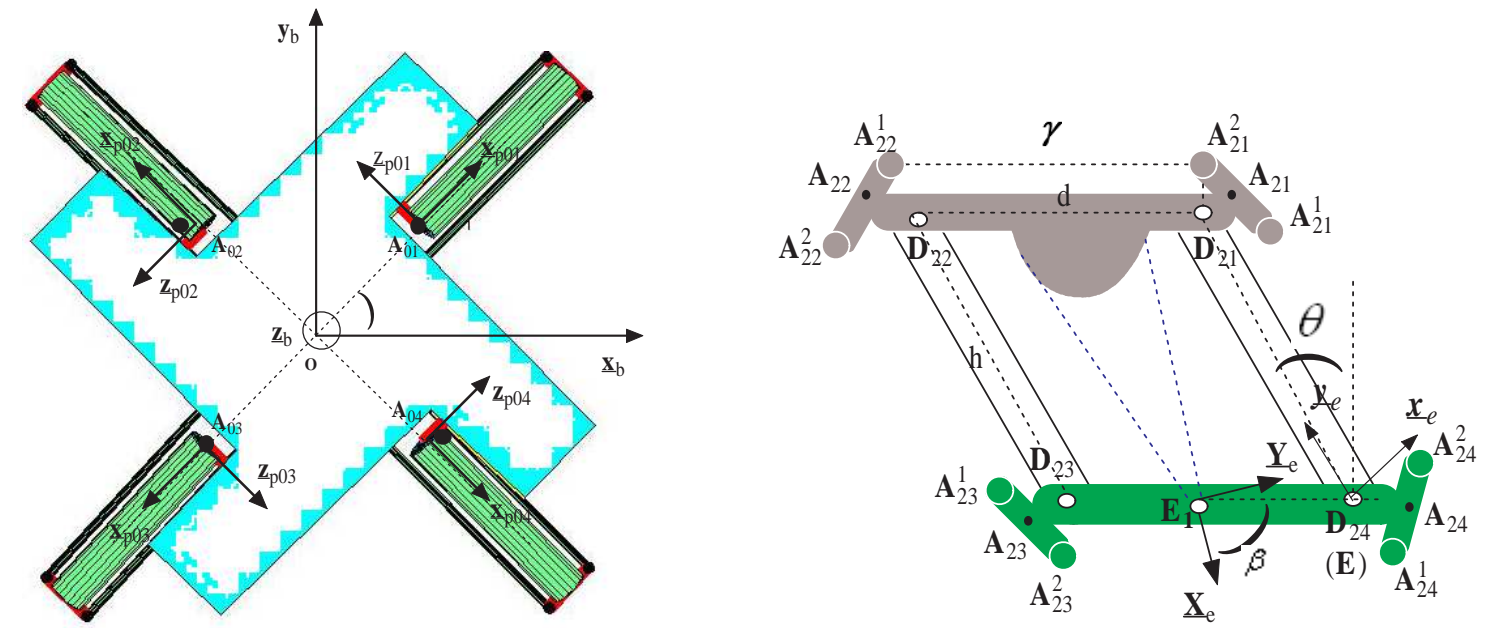

Fig. 9. Top view of the Par4 robot (left) and its nacelle (right).

\section{Contribution to a generic architecture of a broad class of fully parallel robots}

To join together a broad class of fully parallel mechanisms in the same generic architecture, a contribution to a generic structure of the kinematic chains and a generic representation of an articulated nacelle will be discussed in this section. Only the manipulators having the following characteristics will be considered: 
- Fully parallel robot: the number of controlled degrees of freedom of the end-effector is strictly equal to the number of kinematic chains and just one actuator exists in each kinematic chain. ${ }^{5,36}$

- There are only two elements in each kinematic chain. Each element is composed of two rigid bodies linked by a Prismatic joint (fixed or actuated moving joint).

The majority of existing parallel mechanisms are designed with a reduced number of kinematic chain elements compared to serial structures. Since each kinematic chain is usually short and based on the comparative study of some existing parallel robots shown in Section 2, we will restrict the proposed generic architecture to four serial rigid bodies (two serial kinematic elements as defined in Table I) in each kinematic chain. It is suffice from our point of view to describe a broad class of fully parallel robots.

- The joint between two kinematic elements of the kinematic chain must be a Ball, Revolute or Universal joint.

The main reason for this assumption is due to the fact that each kinematic element can be composed of two rigid bodies linked by a Prismatic joint. Consequently, the standard joints (Ball, Revolute, Universal and Prismatic joint) are therefore represented in each kinematic chain.

- The joint between the last element and the nacelle including the moving platform can be a Ball, Revolute or Universal joint.

This is motivated by the fact that a Prismatic joint directly attached to the nacelle is, generally, excluded. An actuated Prismatic joint mounted at or near the fixed base (and not the moving platform) is more interesting for rigidity and high-speed. Additionally, a passive Prismatic joint attached to the nacelle is difficult to control.

- The joint between the first element and the base can be a Revolute or a Fixed joint.

Either joint can ensure rigidity and good kinematic behaviour of the robot because we have introduced enough degrees of freedom between the two kinematic elements (Ball, Revolute or Universal joint), between the last element and the nacelle (Ball, Revolute or Universal joint) and between rigid bodies of each element (Prismatic joint).

- Each kinematic chain of a parallel robot has at least one Prismatic joint which must be a fixed or actuated joint.

A passive Prismatic joint should not be used because it is difficult to control. In addition, a fully parallel robot does not include a kinematic chain with two actuated joints (in some cases, two actuated Prismatic joints).

\subsection{Contribution to generic architecture of the kinematic chains}

Based on the previous assumptions and structures of parallel robots presented in section 2, a representative architecture of a broad class of fully parallel robots is shown in Fig. 10. The articulated nacelle is connected to the base by $n$ kinematic chains. Each chain $\left[\mathbf{A}_{0 i} \mathbf{A}_{2 i}\right]$ is composed of 2 serial kinematics elements $\left[\mathbf{A}_{m i} \mathbf{A}_{(m+1) i}\right],(\mathrm{m}=0 . .1$ and $\mathrm{i}=$ 1..n). In such conditions, this architecture is composed of:

- 4 rigid bodies, in each kinematic chain, $\left[\mathbf{A}_{0 i} \mathbf{P}_{0 i}\right],\left[\mathbf{P}_{0 i} \mathbf{A}_{1 i}\right],\left[\mathbf{A}_{1 i} \mathbf{P}_{1 i}\right]$ and $\left[\mathbf{P}_{1 i} \mathbf{A}_{2 i}\right]$.

- 5 groups of joints shown in Table II: two joints (Prismatic or Fixed joint) at $\mathbf{P}_{m i}$, two joints (Ball, Universal or Revolute joint) at $\mathbf{A}_{1 i}$ and $\mathbf{A}_{2 i}$ and one joint (Revolute or Fixed joint) at $\mathbf{A}_{0 i}$.

The generic architecture in Fig. 10 unifies several families of parallel robots. As shown in Table II, one can easily find the Gough-Stewart platform by taking $l\left(r_{0 i}\right)=0$ (the length of the first element of each kinematic chain) and an Universal joint at $\mathbf{A}_{1 i}=\mathbf{A}_{0 i}$. The Prismatic joint with an axis passing through $\mathbf{P}_{1 i}$ provides the variation of the length $L\left(r_{1 i}\right)$.

Based in the characteristics of several families of parallel robots, following constraints must be satisfied:

- The actuator of each kinematic chain is located at $\mathbf{A}_{0 i}$ or $\mathbf{P}_{0 i}$ or $\mathbf{P}_{1 i}$. The two other passive joints are fixed. 


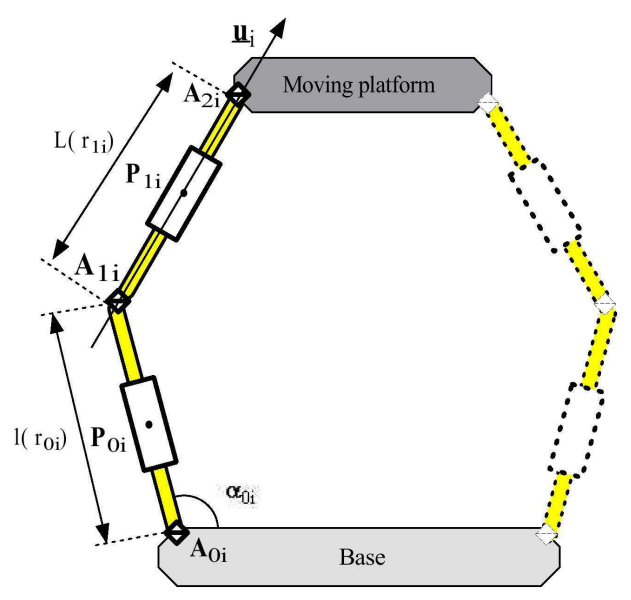

Fig. 10. A generic kinematic chain architecture of a broad class of parallel robots.

This is due to the fact that the actuated joint mounted at or near the fixed base (and not the moving platform) is more interesting for two reasons. First, this configuration reduces the moving platform mass associated with the actuation. Second, it is also a more convenient way to design a parallel robot with low inertia, high load and high speed.

- For each kinematic chain, the actuated joint at $\mathbf{A}_{0 i}$ or $\mathbf{P}_{0 i}$ or $\mathbf{P}_{1 i}$ will be denoted $q_{0 i}$. It may be a Prismatic or a Revolute joint.

Fully parallel robots are designed with only one actuated joint in each kinematic chain.

The conventional actuators are revolute or linear joint mechanisms.

Table II . Illustration of the generic structure.

\begin{tabular}{|c|c|c|}
\hline & Generic Architecture & Gough-Stewart platform \\
\hline $\mathbf{A}_{0 i}$ & Revolute or Fixed joint & Fixed joint \\
\hline $\mathbf{P}_{0 i}$ & Prismatic or Fixed joint & Fixed Prismatic (Fixed) joint \\
\hline $\mathbf{A}_{1 i}$ & Ball or Revolute or Universal joint & Universal joint \\
\hline $\mathbf{P}_{1 i}$ & Prismatic or Fixed joint & Prismatic joint \\
\hline $\mathbf{A}_{2 i}$ & Ball or Revolute or Universal joint & Ball joint \\
\hline$l\left(r_{0 i}\right)$ & $l\left(r_{0 i}\right)$ or constant & constant \\
\hline$L\left(r_{1 i}\right)$ & $L\left(r_{1 i}\right)$ or constant $\neq 0$ & $L\left(r_{1 i}\right)$ \\
\hline \hline & Par4/I4R $/ \mathbf{H 4}$ & Orthoglide $/ \mathbf{I} 4 \mathbf{L}$ \\
\hline $\mathbf{A}_{0 i}$ & Revolute joint & Fixed joint \\
\hline $\mathbf{P}_{0 i}$ & Fixed Prismatic (Fixed) joint & Ball joint \\
\hline $\mathbf{A}_{1 i}$ & Ball joint & Ball joint \\
\hline $\mathbf{P}_{1 i}$ & Fixed Prismatic (Fixed) joint & $l\left(r_{0 i}\right)$ \\
\hline $\mathbf{A}_{2 i}$ & Ball joint & Fixed Print \\
\hline$l\left(r_{0 i}\right)$ & constant & constant \\
\hline$L\left(r_{1 i}\right)$ & constant & \\
\hline
\end{tabular}

Therefore, one can write the actuated joint $q_{0 i}$ as:

$$
q_{0 i}=\lambda_{i} \alpha_{0 i}+\overline{\lambda_{i}}\left(\mu_{i} r_{0 i}+\overline{\mu_{i}} r_{1 i}\right)
$$

where

- $\overline{\mu_{i}}=1-\mu_{i}$ and $\overline{\lambda_{i}}=1-\lambda_{i}$.

- $\mu_{i}=1$ if joint at $\mathbf{P}_{0 i}$ is actuated .

- $\mu_{i}=0$ if joint at $\mathbf{P}_{1 i}$ is actuated . 
- $\lambda_{i}=1$ if joint at $\mathbf{A}_{0 i}$ is actuated .

- $\lambda_{i}=0$ if joint at $\mathbf{P}_{0 i}$ or at $\mathbf{P}_{1 i}$ is actuated .

In the case of an unactuated joint at $\mathbf{P}_{0 i}$ and $\mathbf{P}_{1 i}, \mu_{i}$ can take the value 0 or 1 . This configuration is denoted by $\otimes$. It denotes an indeterminate value.

\subsection{Contribution to a generic representation of a nacelle}

The majority of existing parallel robots are designed with a nacelle composed of one rigid body, which is a compact solid containing the end-effector $\mathbf{E}$ and the moving platform $\mathcal{S}$ (Fig. 11). It is linked to each kinematic chain at $\mathbf{A}_{2 i}$ (e.g. the case of the Gough-Stewart and the Orthoglide robot). The nacelle can be also composed of several elements ${ }^{29} \mathcal{S}_{0 i}$ and $\mathcal{S}$ (Fig. 11). Several research studies have proven that combining a parallel robot and an articulated nacelle (e.g. $\mathrm{H} 4,{ }^{29} \mathrm{I} 4 \mathrm{~L},{ }^{32}$ the $\mathrm{I} 4 \mathrm{R}^{30}$ and the $\mathrm{Par} 4^{31}$ ) is a good alternative for high-speed pick and place operations and obtaining unlimited rotation of the tool around one fixed axis.

In order to propose generic representation of a fully parallel robot nacelle, let us consider the following classifications:

- First family:

This family includes nacelles composed of one rigid body (e.g. Gough-Stewart, Orthoglide $^{34}$, Agile Eye ${ }^{37}$ the Hexapteron ${ }^{38}$ ). This family also contains the nacelle of the fully-isotropic parallel robot. ${ }^{35}$

- Second family:

In this family one can find articulated nacelles of parallel robots with Schoenflies motions $^{39,40}$ (also called 3T1R parallel robots or SCARA motions). The used nacelle is made up of at least 2 articulated bodies (e.g. H4, ${ }^{29} \mathrm{I} 4 \mathrm{~L},{ }^{32}$ the $\mathrm{I} 4 \mathrm{R}^{30}$ and the Par $4^{31}$ ). In general cases, articulated elements of the nacelle are restricted to be coplanar. The nacelle has only translational motion. However, the relative motion of the articulated elements of the nacelle turns the moving platform around an axis of fixed direction, using an amplification system.
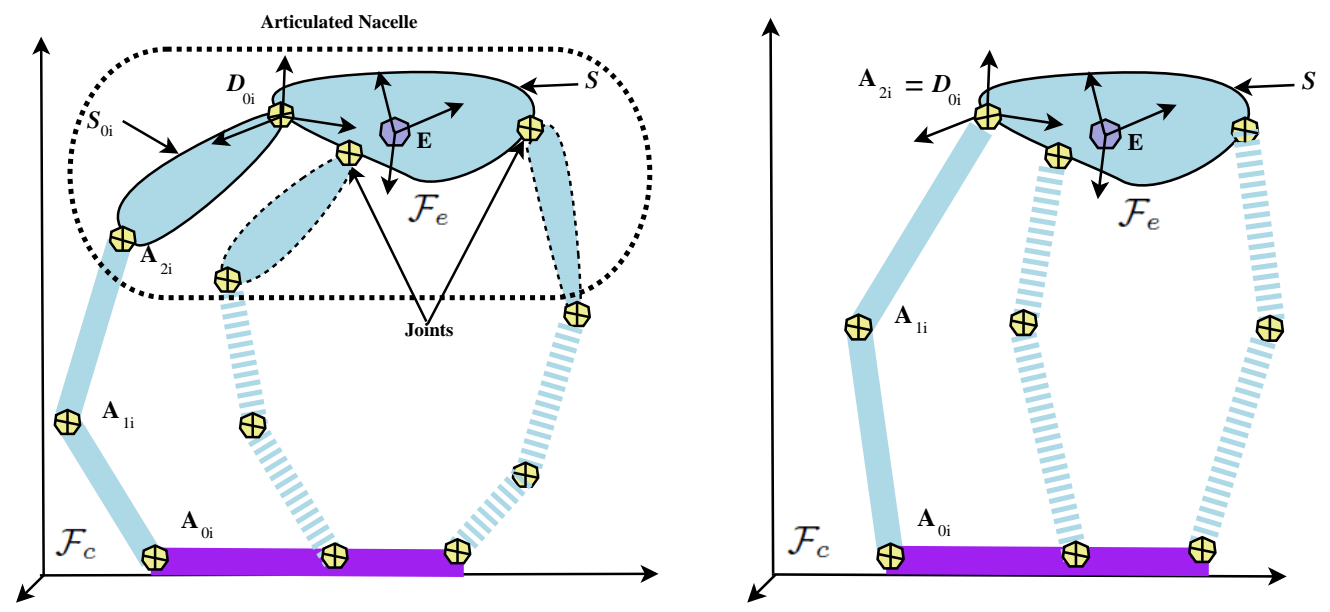

Fig. 11. Generic representation of the articulated nacelle (left) and the nacelle composed of one rigid body (right).

The proposed study is limited to a nacelle made up of n bodies $\mathcal{S}_{0 i}(\mathrm{i}=1 . . \mathrm{n})$ and one central rigid body $\mathcal{S}$. For each kinematic chain $i$, the first element $\mathcal{S}_{0 i}$ contains $\mathbf{A}_{2 i}$ and the second one $\mathcal{S}$ contains the end-effector $\mathbf{E}$. Table III presents nacelles of a broad class of parallel robots according to the generic representation in Fig. 11.

As shown in Fig. 11, one can write:

$$
{ }^{c} \mathbf{A}_{2 i}={ }^{c} \mathbf{E}+{ }^{c} \overrightarrow{\mathbf{E D}_{0 i}}+{ }^{c} \overrightarrow{\mathbf{D}_{0 i} \mathbf{A}_{2 i}}
$$


Table III . Illustration of the generic architecture of the nacelle.

\begin{tabular}{|c|c|c|c|}
\hline The Gough-Stewart platform & & & \\
\hline Kinematic chain i & $\mathcal{S}_{0 i}$ & $\mathcal{S}$ & $\mathbf{D}_{0 i}$ \\
\hline 1 & {$\left[\mathbf{A}_{21} \mathbf{A}_{21}\right]$} & {$\left[\mathbf{A}_{21} \mathbf{E}\right]$} & $\mathbf{A}_{21}$ \\
\hline 2 & {$\left[\mathbf{A}_{22} \mathbf{A}_{22}\right]$} & {$\left[\mathbf{A}_{22} \mathbf{E}\right]$} & $\mathbf{A}_{22}$ \\
\hline 3 & {$\left[\mathbf{A}_{23} \mathbf{A}_{23}\right]$} & {$\left[\mathbf{A}_{23} \mathbf{E}\right]$} & $\mathbf{A}_{23}$ \\
\hline 4 & {$\left[\mathbf{A}_{24} \mathbf{A}_{24}\right]$} & $\mathbf{A}_{24} \mathbf{E}$ & $\mathbf{A}_{24}$ \\
\hline 5 & $\mathbf{A}_{25} \mathbf{A}_{25}$ & $\mathbf{A}_{25} \mathbf{E}$ & $\mathbf{A}_{25}$ \\
\hline 6 & {$\left[\mathbf{A}_{26} \mathbf{A}_{26}\right.$} & {$\left[\mathbf{A}_{26} \mathbf{E}\right]$} & $\mathbf{A}_{26}$ \\
\hline \multicolumn{4}{|l|}{ The H4 or the Par4 robot } \\
\hline Kinematic chain i & $\mathcal{S}_{0 i}$ & $\mathcal{S}$ & $\mathbf{D}_{0 i}$ \\
\hline 1 & {$\left[\mathbf{A}_{21} \mathbf{D}_{21}\right]$} & {$\left[\mathbf{D}_{21} \mathbf{D}_{24}\right.$} & $\mathbf{D}_{21}$ \\
\hline 2 & {$\left[\mathbf{A}_{22} \mathbf{D}_{21}\right]$} & {$\left[\mathbf{D}_{21} \mathbf{D}_{24}\right.$} & $\mathbf{D}_{21}$ \\
\hline 3 & {$\left[\mathbf{A}_{23} \mathbf{D}_{24}\right]$} & {$\left[\mathbf{D}_{24} \mathbf{D}_{21}\right.$} & $\mathbf{D}_{24}$ \\
\hline 4 & {$\left[\mathbf{A}_{24} \mathbf{D}_{24}\right]$} & {$\left[\mathbf{D}_{24} \mathbf{D}_{21}\right.$} & $\mathbf{D}_{24}$ \\
\hline \multicolumn{4}{|l|}{ The I4R robot } \\
\hline Kinematic chain $\mathrm{i}$ & $\mathcal{S}_{0 i}$ & $\mathcal{S}$ & $\mathbf{D}_{0 i}$ \\
\hline 1 & {$\left[\mathbf{A}_{21} \mathbf{D}_{0}\right]$} & {$\left[\mathbf{D}_{0} \mathbf{E}\right]$} & $\mathbf{D}_{0}$ \\
\hline 2 & {$\left[\mathbf{A}_{22} \mathbf{D}_{0}\right]$} & {$\left[\mathbf{D}_{0} \mathbf{E}\right]$} & $\mathbf{D}_{0}$ \\
\hline 3 & {$\left[\mathbf{A}_{23} \mathbf{E}\right]$} & {$\left[\mathbf{E D}_{0}\right]$} & $\mathbf{E}$ \\
\hline 4 & {$\left[\mathbf{A}_{24} \mathbf{E}\right]$} & {$\left[\mathbf{E D}_{0}\right]$} & $\mathbf{E}$ \\
\hline \multicolumn{4}{|l|}{ The I4L robot } \\
\hline Kinematic chain $\mathrm{i}$ & $\mathcal{S}_{0 i}$ & $\mathcal{S}$ & $\mathbf{D}_{0 i}$ \\
\hline 1 & {$\left[\mathbf{A}_{21} \mathbf{D}_{0}\right]$} & {$\left[\mathbf{D}_{0} \mathbf{E}\right]$} & $\mathbf{D}_{0}$ \\
\hline 2 & {$\left[\mathbf{A}_{22} \mathbf{D}_{0}\right]$} & {$\left[\mathbf{D}_{0} \mathbf{E}\right]$} & $\mathbf{D}_{0}$ \\
\hline 3 & {$\left[\mathbf{A}_{23} \mathbf{D}_{1}\right]$} & {$\left[\mathbf{D}_{1} \mathbf{E}\right]$} & $\mathbf{D}_{1}$ \\
\hline 4 & {$\left[\mathbf{A}_{24} \mathbf{D}_{1}\right.$} & {$\left[\mathbf{D}_{1} \mathbf{E}\right]$} & $\mathbf{D}_{1}$ \\
\hline
\end{tabular}

The choice of an appropriate joint between $\mathcal{S}_{0 i}$ and $\mathcal{S}$ centered at $\mathbf{D}_{0 i}$ can be made taking into account the rigidity and a good kinematic behaviour of the parallel mechanism. Thus, a movement of the solid $\mathcal{S}_{0 i}$ must generate a movement of $\mathcal{S}$, at least according to a well-defined axis (the rotation based on the Schoenflies motions). A Prismatic joint has to be avoided, since a movement of $\mathcal{S}_{0 i}$ along the axis of the joint does not move $\mathcal{S}$. Consequently, the rotational movement of $\mathcal{S}_{0 i}$ may only be linear depending on the rotation of the moving platform $\mathcal{S}$ (case of gears, pulley and belt, chain and gears or side by side movement transmissions).

Taking into account the characteristics of the nacelle, one can write in the fixed camera frame $\mathcal{F}_{c}$ :

$$
\left\{\begin{array}{l}
{ }^{c} \mathbf{V}_{\mathbf{D}_{0 i} \in \mathcal{S}}={ }^{c} \mathbf{V}_{\mathbf{D}_{0 i} \in \mathcal{S}_{0 i}} \\
{ }^{c} \boldsymbol{\Omega}_{0 i}=\varrho^{c} \boldsymbol{\Omega}_{e}
\end{array}\right.
$$

where $\varrho$ is the transmission ratio between the two rotational velocities of $\mathcal{S}$ and $\mathcal{S}_{0 i}$.

It should be noted that:

- $\varrho=1$ if the nacelle is representing a single and compact solid $\left({ }^{c} \boldsymbol{\Omega}_{0 i}={ }^{c} \boldsymbol{\Omega}_{e}\right)$.

- $\varrho=0$ if there is no rotational movement of $\mathcal{S}_{0 i}$ with respect to fixed camera frame $\mathcal{F}_{c}$ $\left({ }^{c} \boldsymbol{\Omega}_{0 i}=\mathbf{0}_{3 X 1}\right)$.

Applying the velocity composition law expressed in $\mathcal{F}_{c}$, one can formulate:

$$
\left\{\begin{array}{l}
\frac{d \mathbf{A}_{2 i}}{d t}={ }^{c} \mathbf{V}_{\mathbf{A}_{2 i}}={ }^{c} \mathbf{V}_{\mathbf{D}_{0 i} \in \mathcal{S}_{0 i}}+{ }^{c} \boldsymbol{\Omega}_{0 i} \times{ }^{c} \overrightarrow{\mathbf{D}_{0 i} \mathbf{A}_{2 i}} \\
\frac{d \mathbf{D}_{0 i}}{d t}={ }^{c} \mathbf{V}_{\mathbf{D}_{0 i} \in \mathcal{S}}={ }^{c} \mathbf{V}_{e}+{ }^{c} \boldsymbol{\Omega}_{e} \times{ }^{c} \overrightarrow{\mathbf{E} \mathbf{D}_{0 i}}
\end{array}\right.
$$


From (3) and (4), one can compute:

$$
{ }^{c} \mathbf{V}_{\mathbf{A}_{2 i}}={ }^{c} \mathbf{V}_{e}+{ }^{c} \boldsymbol{\Omega}_{e} \times{ }^{c} \overrightarrow{\mathbf{E D}_{0 i}}+{ }^{c} \boldsymbol{\Omega}_{0 i} \times{ }^{c} \overrightarrow{\mathbf{D}_{0 i} \mathbf{A}_{2 i}}
$$

Consequently, using (3) and (5), one can write the following generic relation:

$$
{ }^{c} \mathbf{V}_{\mathbf{A}_{2 i}}=\left(\mathbf{I}_{3}-\left[{ }^{c} \overrightarrow{\mathbf{E D}_{0 i}}+\varrho^{c} \overrightarrow{\mathbf{D}_{0 i} \mathbf{A}_{2 i}}\right]_{\times}\right){ }^{c} \boldsymbol{\tau}_{e}=\mathbf{G}_{2 i}^{1}{ }^{c} \boldsymbol{\tau}_{e}
$$

where:

$$
\mathbf{G}_{2 i}^{1}=\left(\mathbf{I}_{3}-\left[{ }^{c} \overrightarrow{\mathbf{E D}_{0 i}}+\varrho^{c} \overrightarrow{\mathbf{D}_{0 i} \mathbf{A}_{2 i}}\right]_{\times}\right)
$$

Two significant cases can be deduced:

- The kinematic elements of nacelle $\mathcal{S}$ and $\mathcal{S}_{0 i}$ are rigidly fixed to a single and compact solid (the case of Gough-Stewart platform and the Orthoglide robot), which is the moving platform $(\varrho=1)$. One can write:

$$
{ }^{c} \mathbf{V}_{\mathbf{A}_{2 i}}=\left(\mathbf{I}_{3}-\left[\overrightarrow{\mathbf{E A}_{2 i}}\right]_{\times}\right){ }^{c} \boldsymbol{\tau}_{e}
$$

- There is no rotational movement of $\mathcal{S}_{0 i}$ with respect to fixed camera frame $\mathcal{F}_{c}(\varrho=0)$. However, $\mathcal{S}$ has a rotational movement with respect to fixed camera frame $\mathcal{F}_{c}$ (in the case of the nacelle of H4, Par4, I4R and I4L robots). Consequently, one can write:

$$
{ }^{c} \mathbf{V}_{\mathbf{A}_{2 i}}=\left(\mathbf{I}_{3}-\left[{ }^{c} \overrightarrow{\mathbf{E D}_{0 i}}\right]_{\times}\right){ }^{c} \boldsymbol{\tau}_{e}
$$

\section{Vision-based kinematic using referent edges observation}

The majority of existing parallel mechanisms are designed with slim and cylindrical legs between their base and their moving platform. Thus, one can consider these legs as straight lines for kinematic analysis. ${ }^{5}$ In this section, we will show that referent elements can be observed to extract, directly from the image, the edges used as visual primitives. According to the nature of visual primitives, we will also show that one can have an optimal representation of the state of a parallel robot.

\subsection{Line representation}

Let $\mathcal{L}$ be a $3 \mathrm{D}$ line (Fig. 12). A point-independent representation of this line is the Plücker coordinates $(\underline{\mathbf{u}}, \mathbf{n})^{41}$ (also known as normalized Plücker coordinates since us $\underline{\mathbf{u}}$ is a unit vector), where $\underline{\mathbf{u}}$ is the direction of the line and $\mathbf{n}$ encodes its position. However, noticing that $\mathbf{n}$ is orthogonal to the so-called interpretation plane defined by $\mathcal{L}$ and the origin, one can split it into two parts: the unit vector $\underline{\mathbf{n}}$ defining the interpretation plane and its norm $n$ which is the orthogonal distance of $\mathcal{L}$ to the origin.

\subsection{Projection of the cylindrical referent element in the image}

The closest element $i$ to the nacelle is chosen as the referent element of each kinematic chain. By observing each referent cylindrical element, one can extract the edges $\underline{\mathbf{n}}_{i}^{j}$ with $j=1,2$ associated to the lines' projections in the image plane (Fig. 12). The line's image projection could be represented by the normal vector $\underline{\mathbf{n}}_{i}^{j}(j=1,2)$ to the so-called interpretation plane associated to each edge $j$.

Such a projected line in the image plane, expressed in the camera frame $\mathcal{F}_{c}$, has the following equation:

$$
{ }^{c} \underline{\mathbf{n}}_{i}^{j^{T}{ }^{T}} \mathbf{p}_{i}^{j}=0
$$


where ${ }^{c} \mathbf{p}_{i}^{j}$ represents the coordinates in the camera frame of any point in the image plane, lying on the edge considered as a straight line.
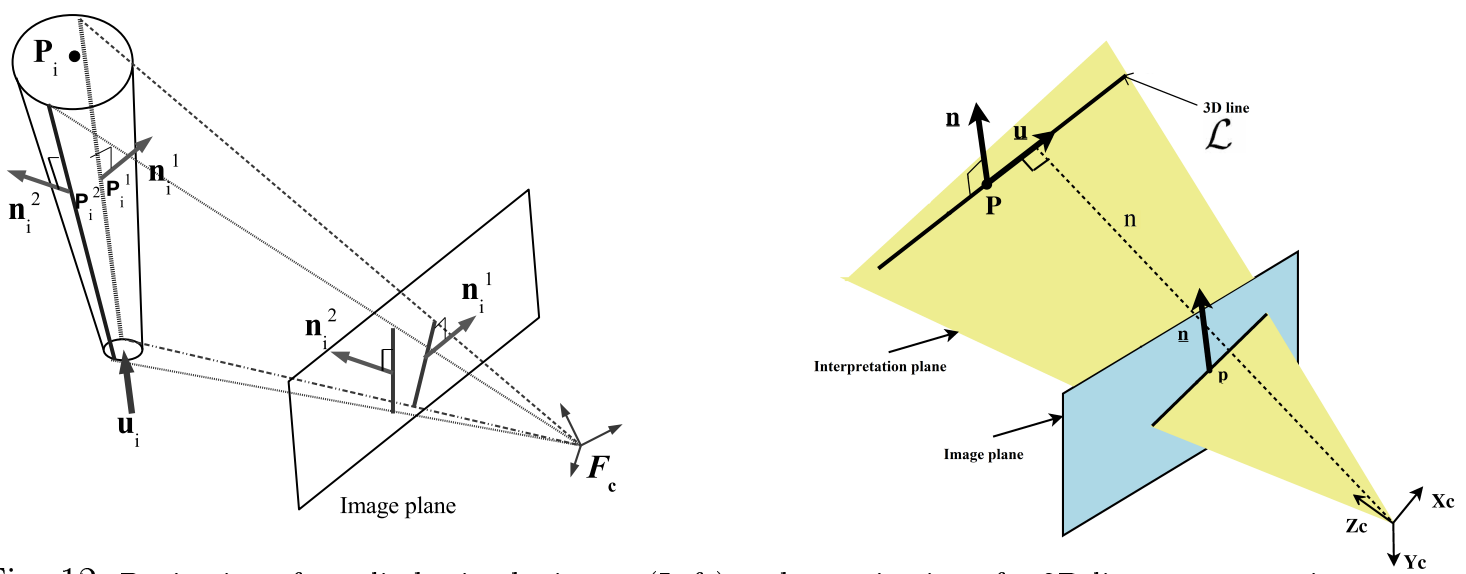

Fig. 12. Projection of a cylinder in the image (Left) and a projection of a 3D line representation.

With the intrinsic camera matrix $\mathbf{K}$, (10) can also be expressed in the image frame as:

$$
{ }^{i m} \underline{\mathbf{n}}_{i}^{j^{T}}{ }^{i m} \mathbf{p}_{i}^{j}={ }^{i m} \underline{\mathbf{n}}_{i}^{j^{T}} \mathbf{K}^{c} \mathbf{p}_{i}^{j}=0
$$

Using (10) and (11), one can easily obtain the conversion from the line equation in the camera frame ${ }^{c} \underline{\mathbf{n}}_{i}^{j}$ to the same conversion in the pixel coordinates ${ }^{i m} \underline{\mathbf{n}}_{i}^{j}$ :

$$
\left\{\begin{array}{l}
{ }^{i m} \underline{\mathbf{n}}_{i}^{j}=\frac{\mathbf{K}^{-T c}{ }^{-\mathbf{n}_{i}^{j}}}{\left\|\mathbf{K}^{-T c} \mathbf{n}_{i}^{j}\right\|} \\
{ }^{c} \underline{\mathbf{n}}_{i}^{j}=\frac{\mathbf{K}^{T i m} \underline{\mathbf{n}}_{i}^{j}}{\left\|\mathbf{K}^{T i m} \underline{\mathbf{n}}_{i}^{j}\right\|}
\end{array}\right.
$$

Consequently, one can deduce the direction ${ }^{c} \underline{\mathbf{u}}_{i}$ of each cylinder ${ }^{42}$ from (12):

$$
{ }^{c} \underline{\mathbf{u}}_{i}=\frac{{ }^{c} \underline{\mathbf{n}}_{i}^{1} \times{ }^{c} \underline{\mathbf{n}}_{i}^{2}}{\left\|{ }^{c} \underline{\mathbf{n}}_{i}^{1} \times{ }^{c} \underline{\mathbf{n}}_{i}^{2}\right\|}
$$

Moreover, consider point $\mathbf{P}_{i}$ lying on the cylinder axis $i$ (Fig. 12), the edge $j$ of each cylinder is defined by the following constraints, expressed in the camera frame:

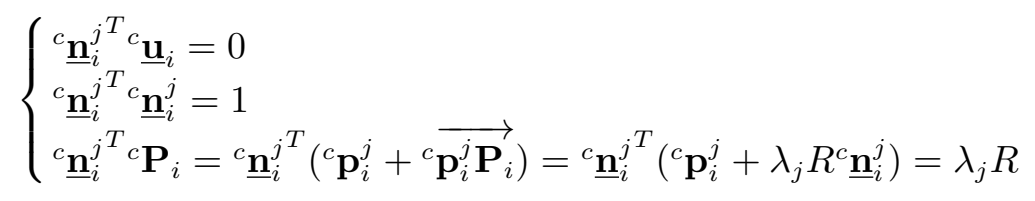

where $\lambda_{1}=\lambda_{2}= \pm 1$ and $R$ is the radius of cylinder $i$.

The last constraint means that the edges are located at a distance $R$ from the cylinder's axis (Fig. 12).

\subsection{The observation of the referent element and the state of a parallel robot}

Two edges can be extracted using cylindrical referent element observation. These edges are directly used in the control loop and in a differential kinematic model.

This reference element is characterized by the reference direction $\underline{\mathbf{u}}_{i}$ and a length $L\left(r_{1 i}\right)$ which can be either variable $L\left(r_{1 i}\right)$ or constant $L\left(r_{1 i}\right)=L$, but never zero. $\mathbf{A}_{1 i}$ and $\mathbf{A}_{2 i}$ are the lower and upper extremities of this element. The lower extremity can be defined using all joint positions of the kinematic chain $\mathbf{A}_{1 i}\left(\alpha_{0 i}, r_{0 i}, \xi_{\text {geom }}\right)$, where $\xi_{\text {geom }}$ is a kinematic parameter. The upper extremity depends on the pose $\mathbf{X}$ of the end-effector $\mathbf{A}_{2 i}\left(\mathbf{X}, \xi_{\text {geom }}\right)$. 
The referent element $\left[\mathbf{A}_{1 i} \mathbf{A}_{2 i}\right]$ of the kinematic chain $i$ can effectively represents the state of parallel robot. Thus, using the constraints (14) applied to point $\mathbf{A}_{2 i}$, one can write the following formula:

$$
\left\{\begin{array}{l}
{ }^{c} \underline{\underline{n}}_{i}^{j^{T}{ }^{c}} \mathbf{A}_{2 i}\left(\mathbf{X}, \xi_{\text {geom }}\right)=\lambda_{i} R \\
{ }^{c} \mathbf{A}_{2 i}={ }^{c} \mathbf{A}_{21}\left(\mathbf{X}, \xi_{\text {geom }}\right)+{ }^{c} \overrightarrow{\mathbf{A}_{21} \mathbf{A}_{2 i}}\left(\mathbf{X}, \xi_{\text {geom }}\right)
\end{array}\right.
$$

The end-effector $\mathbf{X}$ can be represented by the axis-angle representation $\left({ }^{c} \underline{\mathbf{u}}_{e},{ }^{c} \theta_{e}\right)$ of a rotation ${ }^{c} \mathbf{R}_{e}$ and the translation vector ${ }^{c} \mathbf{t}_{e}$. Consequently, one can solve a system with 7 unknown variables represented by ${ }^{c} \underline{\mathbf{u}}_{e},{ }^{c} \theta_{e}$ and ${ }^{c} \mathbf{t}_{e}$ components.

A unique solution of the following system can be computed using constraints in (15) applied to at least 4 kinematic chains:

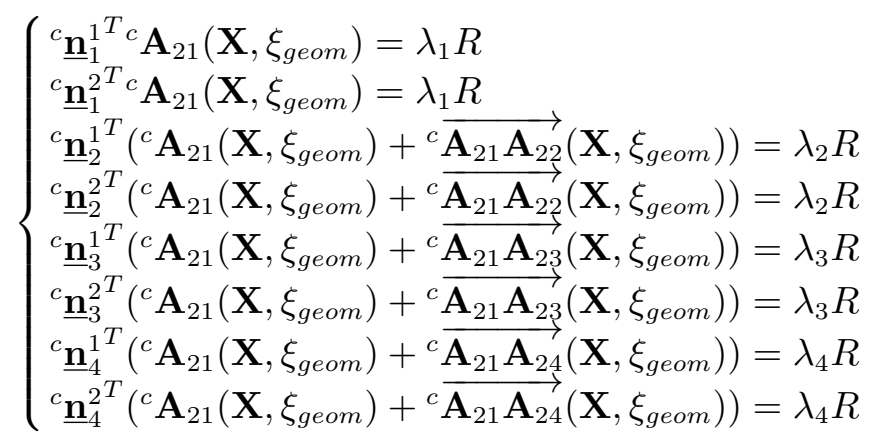

\section{Generic differential inverse kinematic model}

According to the generic architecture presented in section 3, the main objective of this section is to fuse kinematics and projective geometry into a generic projective differential kinematic model for control, which can have the following form:

$$
\left(\begin{array}{c}
\dot{\mathbf{q}} \\
\dot{\dot{\mathbf{u}}} \\
\underline{\mathbf{\mathbf { m }}}
\end{array}\right)=\left(\begin{array}{c}
\mathbf{D}_{e}^{i n v} \\
\mathbf{M}_{e}^{i n v} \\
\mathbf{L}_{e}^{i n v}
\end{array}\right) \boldsymbol{\tau}
$$

where $\mathbf{D}_{e}^{i n v}, \mathbf{M}_{e}^{i n v}$ and $\mathbf{L}_{e}^{i n v}$ are the joint kinematic matrix, the Cartesian kinematic matrix associated to the directions and the Cartesian kinematic matrix associated to the edges, respectively.

The kinematic chains' closure around the reference element $\left[\mathbf{A}_{1 i} \mathbf{A}_{2 i}\right]$ (Fig. 10) yields, for each kinematic chain $i$, the following kinematic model in generic vector form:

$$
L\left(r_{1 i}\right) \underline{\mathbf{u}}_{i}=\overrightarrow{\mathbf{A}_{1 i} \mathbf{A}_{2 i}}=\mathbf{A}_{2 i}\left(\mathbf{X}, \xi_{\text {geom }}\right)-\mathbf{A}_{1 i}\left(\alpha_{0 i}, r_{0 i}, \xi_{\text {geom }}\right)
$$

where $\mathbf{X}$ is a representation of the end-effector pose.

Assuming that the kinematic parameters $\xi_{\text {geom }}$ are constants, time differentiating of the kinematic model (18) gives:

$$
\begin{aligned}
& \dot{L}\left(r_{1 i}\right) \underline{\mathbf{u}}_{i}+L\left(r_{1 i}\right) \underline{\mathbf{u}}_{i}= \\
& \frac{d}{d t}\left(\mathbf{A}_{2 i}\left(\mathbf{X}, \xi_{\text {geom }}\right)\right)-\frac{d}{d t}\left(\mathbf{A}_{1 i}\left(\alpha_{0 i}, r_{0 i}, \xi_{\text {geom }}\right)\right)
\end{aligned}
$$


Time differentiating of $L\left(r_{1 i}\right), \mathbf{A}_{2 i}$ and $\mathbf{A}_{1 i}$ provides:

$$
\left\{\begin{array}{l}
\frac{d}{d t}\left(L\left(r_{1 i}\right)\right)=\frac{\partial L\left(r_{1 i}\right)}{\partial r_{1 i}} \dot{r}_{1 i} \\
\frac{d}{d t}\left(\mathbf{A}_{2 i}\right)=\frac{\partial \mathbf{A}_{2 i}}{\partial \mathbf{X}} \mathcal{L}_{X} \boldsymbol{\tau} \\
\frac{d}{d t}\left(\mathbf{A}_{1 i}\right)=\frac{\partial \mathbf{A}_{1 i}}{\partial \alpha_{0 i}} \dot{\alpha}_{0 i}+\frac{\partial \mathbf{A}_{1 i}}{\partial r_{0 i}} \dot{r}_{0 i}
\end{array}\right.
$$

where $\mathcal{L}_{X}$ is the matrix relating time differentiating of the Cartesian pose to the Cartesian velocity. ${ }^{4}$

One can rewrite (19) as:

$$
L\left(r_{1 i}\right) \underline{\dot{\mathbf{u}}}_{i}=\mathbf{G}_{2 i}^{1} \boldsymbol{\tau}+\mathbf{G}_{1 i}^{0} \dot{\alpha}_{0 i}+\mathbf{G}_{1 i}^{1} \dot{r}_{0 i}+\mathbf{G}_{1 i}^{2} \dot{r}_{1 i}
$$

where

- $\mathbf{G}_{2 i}^{1}=\frac{\partial \mathbf{A}_{2 i}}{\partial \mathbf{X}} \mathcal{L}_{X}$ is the interaction matrix associated to the 3D point $\mathbf{A}_{2 i}$ expressed in (7) (see Section. 3.2 for more details).

- $\mathbf{G}_{1 i}^{0}=-\frac{\partial \mathbf{A}_{1 i}}{\partial \alpha_{0 i}}, \mathbf{G}_{1 i}^{1}=-\frac{\partial \mathbf{A}_{1 i}}{\partial r_{0 i}}$ and $\mathbf{G}_{1 i}^{2}=-\frac{\partial L\left(r_{1 i}\right)}{\partial r_{1 i}} \underline{\mathbf{u}}_{i}$ are joint kinematic matrices.

5.1. Differential inverse kinematic model associated to the actuated joints

In the case of a parallel robot with one actuated joint $q_{0 i}$ in each kinematic chain, the vector of the joint velocities $\dot{\mathbf{q}}$ is obtained from the individual joint velocity $\dot{q}_{0 i}$. The differential inverse kinematic model associated to all actuated joints expressed in the camera frame $\mathcal{F}_{c}$ can have the following formula:

$$
\dot{\mathbf{q}}=\mathbf{D}_{e}^{i n v c} \boldsymbol{\tau}_{e}
$$

Assuming that only one joint variable $\left(\alpha_{0 i}, r_{0 i}\right.$ or $\left.r_{1 i}\right)$ is used in $(21)$, one can write:

$$
\begin{aligned}
& L\left(r_{1 i}\right)^{c} \dot{\mathbf{u}}_{i}= \\
& \mathbf{G}_{2 i}^{1 c} \boldsymbol{\tau}_{e}+\mathbf{G}_{1 i}^{0} \dot{\alpha}_{0 i}+\mathbf{G}_{1 i}^{1} \dot{r}_{0 i}+\mathbf{G}_{1 i}^{2} \dot{r}_{1 i} \equiv \mathbf{G}_{2 i}^{1}{ }^{c} \boldsymbol{\tau}_{e}+\mathbf{G}_{1 i} \dot{q}_{0 i}
\end{aligned}
$$

where $\mathbf{G}_{1 i}$ depends on $q_{0 i}$ (see section 3.1 and (1)) and has the following generic equation:

$$
\mathbf{G}_{1 i}=\lambda_{i} \mathbf{G}_{1 i}^{0}+\overline{\lambda_{i}}\left(\mu_{i} \mathbf{G}_{1 i}^{1}+\overline{\mu_{i}} \mathbf{G}_{1 i}^{2}\right)
$$

Since ${ }^{c} \underline{\mathbf{u}}_{i}^{T c} \underline{\mathbf{u}}_{i}=0,(23)$ gives the differential inverse kinematic model associated to $q_{0 i}$ :

$$
\dot{q}_{0 i}=-\frac{{ }^{c} \underline{\mathbf{u}}_{i}^{T} \mathbf{G}_{2 i c}^{1}}{{ }^{{ }^{\mathbf{u}}} \underline{\underline{i}}_{i}^{T} \mathbf{G}_{1 i}} \boldsymbol{\tau}_{e}=\mathbf{D}_{e i}^{i n v c} \boldsymbol{\tau}_{e}
$$

where $\mathbf{D}_{e i}^{i n v}$ is the individual matrix (row vector) of $\mathbf{D}_{e}^{i n v}$.

\subsection{Differential inverse kinematic model associated to directions}

Using (23) and (25), the differential inverse kinematic model associated to directions $\underline{\mathbf{u}}_{i}$ can be written as:

$$
{ }^{c} \underline{\dot{\mathbf{u}}}_{i}=\frac{1}{L\left(r_{1 i}\right)}\left(\mathbf{I}_{3}-\frac{\mathbf{G}_{1 i}{ }^{c} \underline{\mathbf{u}}_{i}^{T}}{{ }^{c} \underline{\mathbf{u}}_{i}^{T} \mathbf{G}_{1 i}}\right) \mathbf{G}_{2 i}^{1}{ }^{c} \boldsymbol{\tau}_{e}=\mathbf{M}_{i}{ }^{c} \boldsymbol{\tau}_{e}
$$

where $\mathbf{M}_{i}=\frac{1}{L\left(r_{1 i}\right)}\left(\mathbf{I}_{3}-\frac{\mathbf{G}_{1 i}{ }^{c} \mathbf{u}_{i}^{T}}{{ }^{c} \underline{\underline{u}}_{i}^{T} \mathbf{G}_{1 i}}\right) \mathbf{G}_{2 i}^{1}$ is the individual matrix of $\mathbf{M}_{e}^{i n v}$. 
5.3. Differential inverse kinematic model associated to edges

The time derivative of constraints in (14) applied to point $\mathbf{A}_{2 i}$ induces a first differential inverse kinematic model associated to the edges expressed in the camera frame and has the following equation: ${ }^{20}$

$$
{ }^{c} \underline{\dot{\mathbf{n}}}_{i}^{j}=\left(\mathbf{R}_{1 i}^{j} \mathbf{G}_{2 i}^{1}+\mathbf{R}_{2 i}^{j} \mathbf{M}_{i}\right) \boldsymbol{\tau}={ }^{c} \mathbf{L}_{i}^{j c} \boldsymbol{\tau}_{e}
$$

where

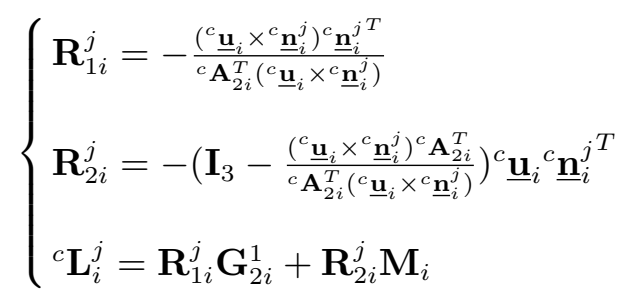

where $\mathbf{M}_{i}$ is defined in (26) and $\mathbf{G}_{2 i}^{1}$ is defined in (7).

This proves that the movement of the platform depends linearly on the variation of the movement of $\mathbf{A}_{2 i}$ represented by $\mathbf{G}_{2 i}^{1}$ and on the differential inverse kinematic model associated to directions $\mathbf{M}_{i}$.

A second differential inverse kinematic model associated to edges expressed in the image frame can also be computed. As a results one needs to present the interaction matrix ${ }^{i m} \mathbf{L}_{i}^{j}$ relating the Cartesian velocity ${ }^{c} \boldsymbol{\tau}_{e}$ to the time differentiating of the edge vector of each referent element ${ }^{i m} \underline{\underline{\dot{\mathbf{n}}}}_{i}^{j}$ expressed in the image frame:

$$
{ }^{i m} \underline{\underline{\mathbf{n}}}_{i}^{j}={ }^{i m} \mathbf{L}_{i}^{j c} \boldsymbol{\tau}_{e}
$$

where ${ }^{i m} \mathbf{L}_{i}^{j}$ is the individual matrix of $\mathbf{L}_{e}^{i n v}$.

${ }^{i m} \mathbf{L}_{i}^{j}$ can be written as a product of two matrices:

$$
{ }^{i m} \mathbf{L}_{i}^{j}={ }^{i m} \mathbf{J}_{c}{ }^{c} \mathbf{L}_{i}^{j}
$$

where ${ }^{i m} \mathbf{J}_{c}$ is associated to the camera-to-pixel change of frame:

$$
{ }^{i m} \underline{\dot{\mathbf{n}}}_{i}^{j}={ }^{i m} \mathbf{J}_{c}{ }^{c} \underline{\dot{\mathbf{n}}}_{i}^{j}
$$

According to ref. [20], ${ }^{i m} \mathbf{J}_{c}$ can be written as:

$$
{ }^{i m} \mathbf{J}_{c}=\left\|\mathbf{K}^{T i m} \underline{\mathbf{n}}_{i}^{j}\right\|\left(\mathbf{I}_{3}-{ }^{i m} \underline{\mathbf{n}}_{i}^{j i m} \underline{\mathbf{n}}_{i}^{j^{T}}\right) \mathbf{K}^{-T}
$$

\section{Visual servoing using referent elements observation}

\subsection{Error to servo}

The generic control law is based on the observation of referent elements and the extraction of the visual primitives $\mathbf{s}_{i}^{j}={ }^{i m} \underline{\mathbf{n}}_{i}^{j}$ used for regulation. We choose to minimize the error $\mathbf{e}_{i}^{j}$ between the edge in the current position ${ }^{i m} \underline{\mathbf{n}}_{i}^{j}$ and the edge in a desired position ${ }^{i m} \underline{\mathbf{n}}_{i}^{j *}$. First, the control law gives the Cartesian velocity according to the error. The differential inverse kinematic model associated to the joints will then provide the joint velocities.

The error to servo ${ }^{20,21}$ is:

$$
\mathbf{e}_{i}^{j}={ }^{i m} \underline{\mathbf{n}}_{i}^{j} \times{ }^{i m} \underline{\mathbf{n}}_{i}^{j^{*}}
$$

Taking into account that the proposed generic architecture is composed of $n$ kinematic chains, the vector of all errors is $\mathbf{e}=\left(\mathbf{e}_{1}^{1^{T}} \mathbf{e}_{1}^{2^{T}} \ldots \mathbf{e}_{n}^{1^{T}} \mathbf{e}_{n}^{2^{T}}\right)^{T}$. 
6.2. Generic control law

Time derivative of (33) gives:

$$
\dot{\mathbf{e}}_{i}^{j}={ }^{i m} \underline{\mathbf{\underline { \mathbf { n } }}}_{i}^{j} \times{ }^{i m} \underline{\mathbf{n}}_{i}^{j^{*}}=-\left[{ }^{i m} \underline{\mathbf{n}}_{i}^{j^{*}}\right]_{\times}^{i m} \underline{\underline{\mathbf{n}}}_{i}^{j}
$$

Using (29), one can compute:

$$
\dot{\mathbf{e}}_{i}^{j}=\mathbf{N}_{i}^{j c} \boldsymbol{\tau}_{e}
$$

where

$$
\mathbf{N}_{i}^{j}=-\left[{ }^{i m} \underline{\mathbf{n}}_{i}^{j^{*}}\right]_{\times}{ }^{i m} \mathbf{L}_{i}^{j}
$$

Taking into account an exponential behaviour of the error $\left(\dot{\mathbf{e}}=-\lambda_{p} \mathbf{e}\right),(35)$ gives:

$$
{ }^{c} \boldsymbol{\tau}_{e}=-\lambda_{p} \widehat{\mathbf{N}}^{+} \mathbf{e}
$$

where $\mathbf{N}$ is a compound matrix from the associated individual interaction matrices $\mathbf{N}_{i}^{j}$ and $\lambda_{p}$ is a constant parameter.

Inserting (37) into (22) delivers the final generic control law:

$$
\dot{\mathbf{q}}=-\lambda_{p} \widehat{{ }^{c} \mathbf{D}_{e}^{i n v}} \widehat{\mathbf{N}}^{+} e
$$

\section{Experimental validations}

\subsection{Experimental context}

The proposed approaches are validated on the Gough-Stewart Platform and the Par4 robot. By providing an interface with Linux-RTAI, the kinematic control of the GoughStewart platform and the Par4 robot is ensured using ViSP library ${ }^{43}$ for extracting edges, tracking and matrix computation. Each robot is observed by a perspective camera (1024x780 pixels, IEEE1394) fixed with respect to the base reference frame. The camera is placed in front of the robot legs so that it can cover the robot workspace (Fig. 13 and Fig. 14).
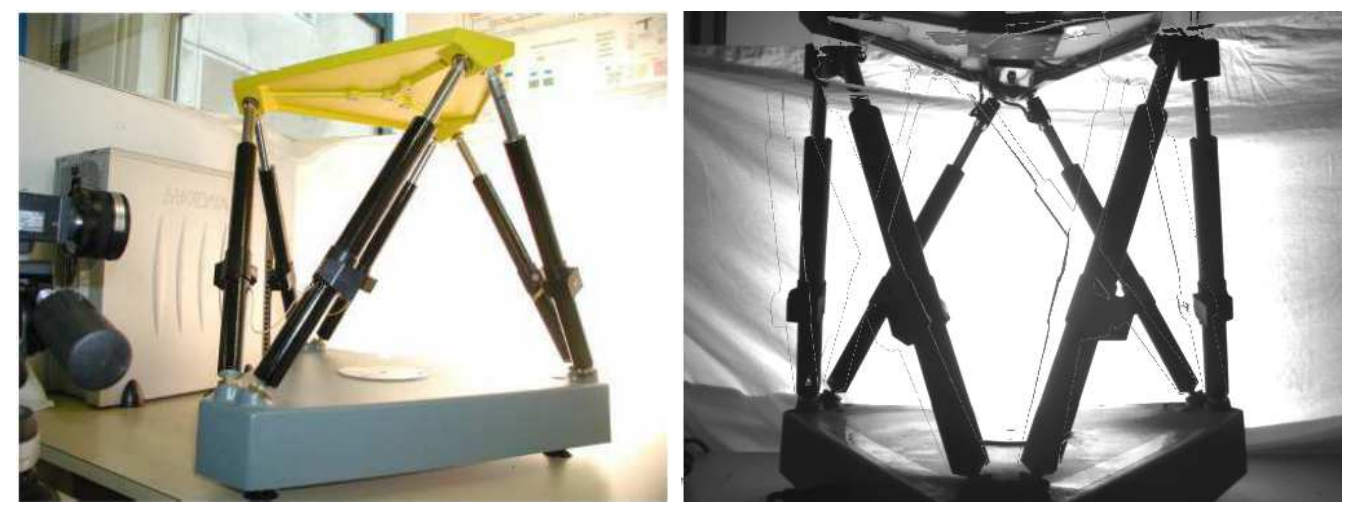

Fig. 13. A photograph of a Gough-Stewart platform (left) and a current configuration seen from the camera (the desired configuration is in the background).

\subsection{Experimental validation on a Gough-Stewart Platform}

7.2.1. The Gough-Stewart platform model according to the generic structure model. In the case of the Gough-Stewart platform, the two extremities of the referent element are $\mathbf{A}_{1 i}$ and $\mathbf{A}_{2 i}$ (see Fig. 3 and Fig. 10). Notice that $\mathbf{A}_{1 i}$ is a constant parameter $\left(\frac{d\left({ }^{c} \mathbf{A}_{1 i}\right)}{d t}=\mathbf{0}\right)$. 


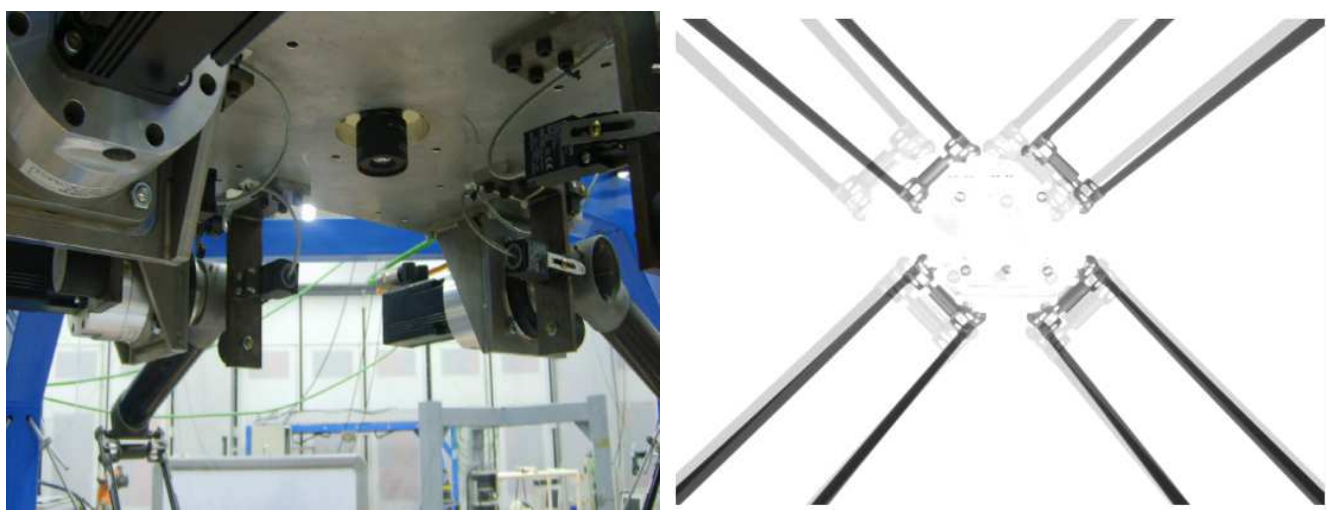

Fig. 14. A photograph of the Par4 robot (left) and a current configuration seen from the camera (the desired configuration is in the background).

The length of the referent element $\left[\mathbf{A}_{1 i} \mathbf{A}_{2 i}\right]$ is $L\left(r_{1 i}\right)=r_{1 i}$. The kinematic model in vector form (18) can be written as:

$$
r_{1 i}{ }^{c} \underline{\mathbf{u}}_{i}={ }^{c} \mathbf{A}_{2 i}-{ }^{c} \mathbf{A}_{1 i}
$$

Table IV gives the characteristics of the Gough-Stewart platform models deduced from the proposed generic model (see section 3 and section 5).

Table IV . The Gough-Stewart models.

\begin{tabular}{|c|c|}
\hline Actuators in each kinematic chain & 1 \\
\hline$\mu_{i}$ & 0 \\
\hline$\lambda_{i}$ & 0 \\
\hline$q_{0 i}$ & $r_{1 i}$ \\
\hline $\mathbf{A}_{1 i}$ & $\mathbf{A}_{1 i}=\mathbf{A}_{0 i}=\mathbf{P}_{0 i}$ \\
\hline$\varrho$ & 1 \\
\hline Nacelle & $\mathbf{0}$ \\
\hline $\mathbf{G}_{1 i}^{0}$ & $\mathbf{0}$ \\
\hline $\mathbf{G}_{1 i}^{1}$ & $-{ }^{c} \underline{\mathbf{u}}_{i}$ \\
\hline $\mathbf{G}_{1 i}^{2}$ & $-{ }^{2} \underline{\mathbf{u}}_{i}$ \\
\hline $\mathbf{G}_{1 i}$ & $\left(\mathbf{I}_{3}-{ }^{c}\left[\mathbf{E}_{\overline{\mathbf{E}}} \mathbf{A}_{2 i}\right]_{\times}\right)$ \\
\hline $\mathbf{G}_{2 i}^{1}$ & \\
\hline
\end{tabular}

7.2.2. Image-based visual servoing results. To evaluate the control approach, the GoughStewart platform is asked to reach a desired pose (Fig. 13) obtained from an initial configuration. Therefore, Fig. 15 presents the evolution of the unit-less errors in the image and the Cartesian velocities.

The errors converge exponentially to 0 with a perfect decoupling from an initial position to the desired one. The convergence errors are essentially due to the accuracy level of the edges' extraction. The position of the vision sensor (Fig. 13) does not allow the same precision on all referent kinematic elements, which generates measurement sensitivity.

\subsection{Experimental validation on the Par4 robot}

7.3.1. The Par4 model according to the generic structure model. As shown in Fig. 6 , each forearm $i$ consists of two kinematic elements $\left[\mathbf{A}_{1 i}^{1} \mathbf{A}_{2 i}^{1}\right]$ and $\left[\mathbf{A}_{1 i}^{2} \mathbf{A}_{2 i}^{2}\right]$. We choose to observe only the first referent element $\left[\mathbf{A}_{1 i}^{1} \mathbf{A}_{2 i}^{1}\right]$ with length $L\left(r_{1 i}\right)=L$ (see Fig. 6 and Fig. 10).

The kinematic model (18) can be written as:

$$
L^{c} \underline{\mathbf{u}}_{i}={ }^{c} \mathbf{A}_{2 i}^{1}-{ }^{c} \mathbf{A}_{1 i}^{1}
$$



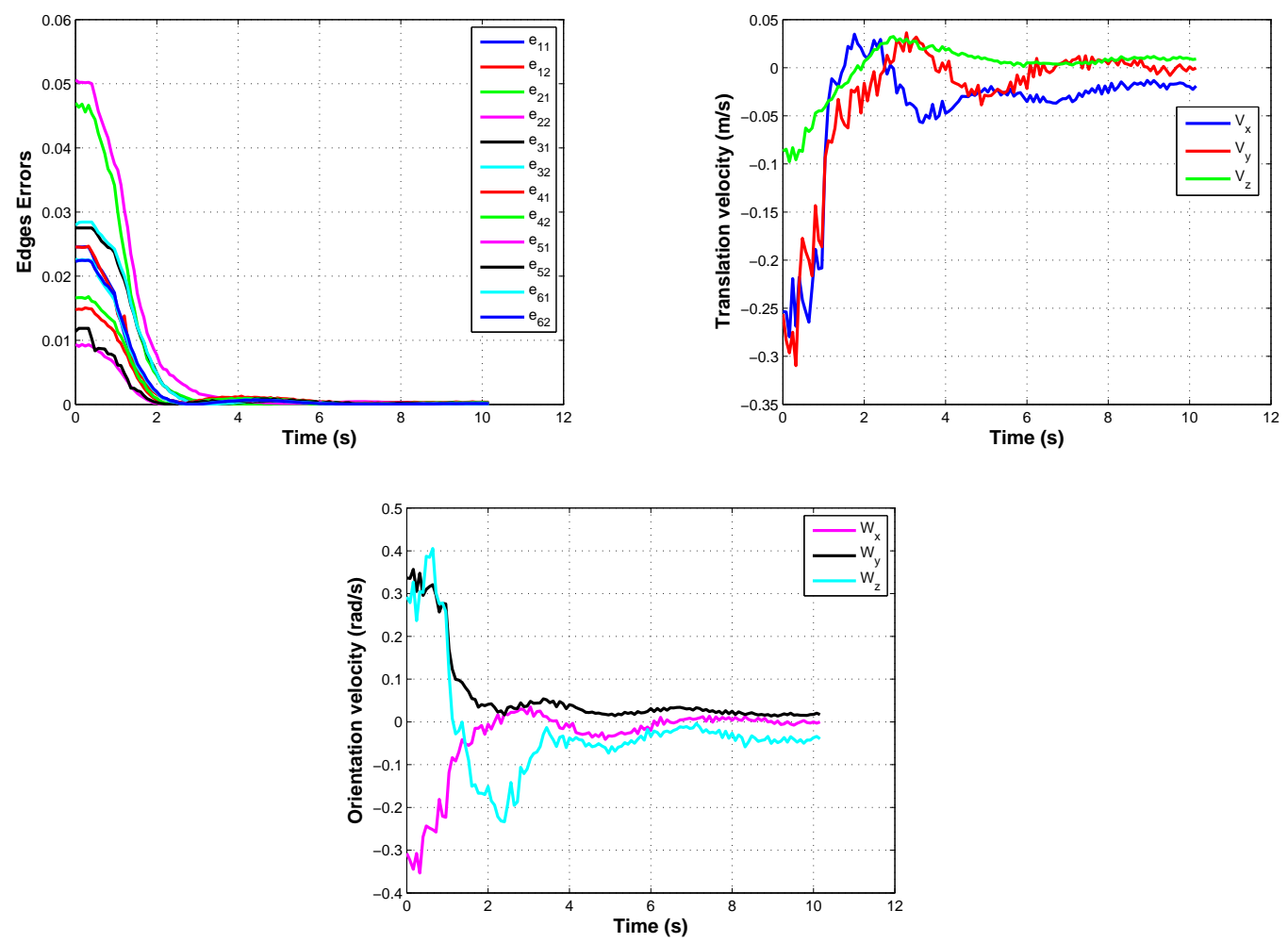

Fig. 15. Visual servoing of the Gough-Stewart platform: edge errors and Cartesian velocity.

The direction ${ }^{c} \underline{\mathbf{x}}_{p 0 i}$ of each arm is a unit vector. Each arm has a rotational movement around ${ }^{c} \underline{\mathbf{z}}_{p 0 i}$, with angle $q_{0 i}=\alpha_{0 i}$.

It should be noted that:

- ${ }^{c} \mathbf{A}_{0 i}$ and ${ }^{c} \overrightarrow{\mathbf{A}_{1 i}^{2} \mathbf{A}_{1 i}^{1}}=-H^{c} \underline{\mathbf{Z}}_{p 0 i}$ are constants.

- $l\left(r_{0 i}\right)=l$.

- ${ }^{c} \mathbf{A}_{1 i}^{1}={ }^{c} \mathbf{A}_{0 i}+l{ }^{c} \underline{\mathbf{x}}_{p 0 i}+\frac{1}{2} c \overrightarrow{\mathbf{A}_{1 i}^{2} \mathbf{A}_{1 i}^{1}}$.

One can compute:

$$
\frac{d\left({ }^{c} \mathbf{A}_{1 i}^{1}\right)}{d t}=l \frac{d}{d t}\left({ }^{c} \underline{\mathbf{x}}_{p 0 i}\right)=\dot{q}_{0 i} l^{c} \underline{\mathbf{y}}_{p 0 i}
$$

where ${ }^{c} \underline{\mathbf{y}}_{p 0 i}={ }^{c} \underline{\mathbf{z}}_{p 0 i} \times{ }^{c} \underline{\mathbf{x}}_{p 0 i}$

Table $\mathrm{V}$ presents the Par4 models according to the generic model (see section 3 and section 5).

7.3.2. Experimental results. The par4 robot is asked to reach the desired pose (Fig. 14) obtained from the initial configuration by a translation along the three axes and a rotation around the ${ }^{c} \underline{\mathbf{z}}$ axis. Fig. 16 shows that the edge errors and the Cartesian velocities converge to 0 . An experimental test was performed using approximately 15-20 FPS, IEEE1394 camera. This is largely sufficient to prove the validity of the proposed approaches. As shown in Fig. 16, one can see that the maximum speed is approximately equal to 0.012 $\mathrm{m} / \mathrm{s}$ in translation and $0.042 \mathrm{rad} / \mathrm{s}$ in orientation. Notice that one can use a high speed camera (e.g. Rolling Shutter Camera) to achieve a high-speed vision-based control ${ }^{44}$ and to improve speed and acceleration values. Recently, the real-time vision-based control, the analysis and the real-time trajectory tracking control validated on a real parallel robots, e.g. ${ }^{45-47}$, have attracted more interests. 
Table V . The Par4 models.

\begin{tabular}{|c|c|}
\hline Actuators in each kinematic chain & 1 \\
\hline$\mu_{i}$ & 0 or 1 \\
\hline$\lambda_{i}$ & 1 \\
\hline$q_{0 i}$ & $\alpha_{0 i}$ \\
\hline $\mathbf{A}_{1 i}$ & $\mathbf{A}_{1 i}^{1}$ \\
\hline$\varrho$ & 0 \\
\hline Nacelle & $-l^{l} \underline{\mathbf{y}}_{p 0 i}$ \\
\hline $\mathbf{G}_{1 i}^{0}$ & $\mathbf{0}$ \\
\hline $\mathbf{G}_{1 i}^{1}$ & $\mathbf{0}$ \\
\hline $\mathbf{G}_{1 i}^{2}$ & $-l^{c} \underline{\mathbf{y}}_{p 0 i}$ \\
\hline $\mathbf{G}_{1 i}$ & $\boldsymbol{\Omega}_{0 i}=0$ \\
\hline $\mathbf{G}_{2 i}^{1}$ & $\left(\mathbf{I}_{3}-\left[{ }^{c} \overrightarrow{\mathbf{D}_{24} \mathbf{D}_{0 i}}\right]_{\times}\right)$ \\
\hline
\end{tabular}
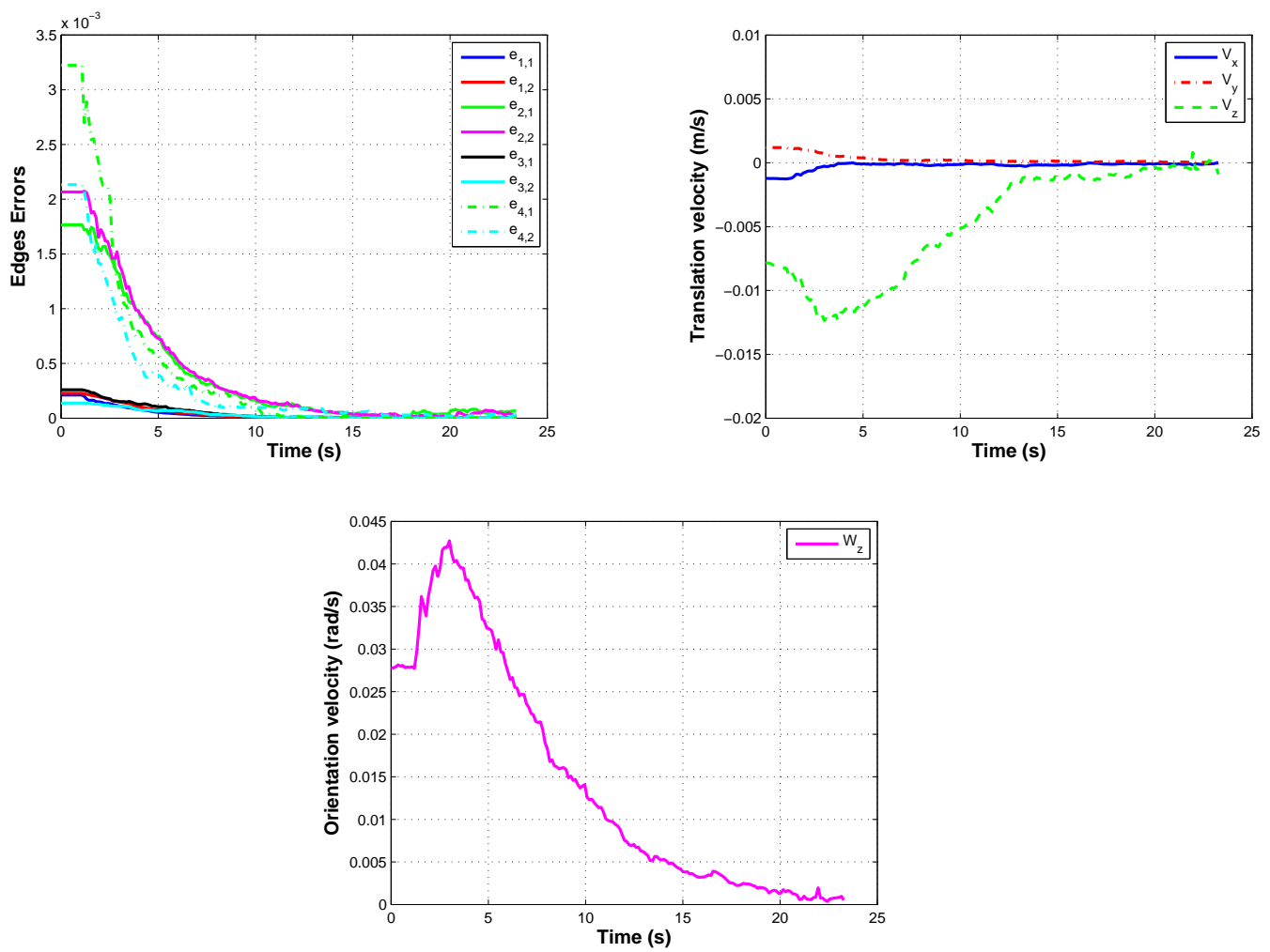

Fig. 16. Visual servoing of Par4 robot: edges errors and Cartesian velocity.

In the kinematic modeling of the Par4 robot shown in Table $\mathrm{V}$, one needs to estimate points $\mathbf{A}_{1 i}^{1}$. The first option is to use redundant sensors to measure $q_{0 i}=\alpha_{0 i}$ and to compute ${ }^{c} \mathbf{A}_{1 i}^{1}={ }^{c} \mathbf{A}_{0 i}+l^{c} \underline{\mathbf{x}}_{p 0 i}+\frac{1}{2} c \overrightarrow{\mathbf{A}_{1 i}^{2} \mathbf{A}_{1 i}^{1}}$. This method is not always technically feasible. It has been shown in ref. [48] that these parameters can be measured using only vision in the case of the I4R robot. Moreover, it was shown that one can get rid of the joint values in the same movement. In this paper, the same method is used in the case of Par4 robot. The edges and constraints (14) deriving from the image projection of the cylindrical referent elements are used to measure ${ }^{c} \underline{\mathbf{u}}_{i}$ and $\mathbf{A}_{2 i}^{1}$. Finally, ${ }^{c} \mathbf{A}_{1 i}^{1}$ can then be deduced from (40) without proprioceptive sensors. 


\section{Discussion}

It should be noted that the observation of the cylindrical referent kinematic elements by a single perspective camera causes a self-occlusion problem since the legs in the background of the image may be hidden by those in the foreground (Fig. 13). Moreover, the perspective projection of some referent elements appears nearly parallel in the image, which is a noise sensitive configuration for the direction extraction. In ref. [44], a way to solve the occlusion problem is to turn to an observable portion of the line representing the cylindrical leg edge which can be enough for visual tracking. Omnidirectional camera can also overcome these problems since it provides a 360 degree field of view of the surroundings. Placed between the legs, it provides a more convenient observation of the lines. ${ }^{49,50}$ An alternative solution is to observe only the $m_{n}$ observable legs among the $n$ legs of the parallel robot $\left(m_{n}<n\right) .{ }^{19}$ As shown in ref. [26], the minimal number of observed legs $\left(m_{n}\right)$ should be, for $3 \mathrm{D}$ unit orientations vectors, an integer greater than $n / 2$.

Let us now consider the case of a referent kinematic element having another shape such as a parallelepiped, a portion of a cone, square pyramid or cuboid. Different points can be discussed here. The first step is to choose the visual primitives (edges, lines, faces) and the vision system (perspective, omnidirectional or stereo camera) to use in the control. Afterwards, the constraints in Section 4 (Eqs. (10), (13) and (14)) should be modified and adjusted to fit each shape and can then be used to compute the direction and the differential inverse kinematic model associated to visual primitives. In some cases, different edges and faces of shapes are not observable. Different techniques like shape detection or tracking using contours can be used to avoid this problem.

The previously mentioned issues can be the subject of several future studies. Once these points are studied, they can improve our approach and make it even more generic.

\section{Conclusion}

This paper presented a generic modeling and vision-based control of a broad class of parallel robots designed with cylindrical legs and one actuated joint. A generic architecture composed of a generic representation of the kinematic chains and the nacelle was first introduced.

Based on the geometric specifications of each kinematic chain and the nacelle, a generic projective kinematic model was examined. Inspired by the geometry of lines, this model relies on the kinematic specificity of the last kinematic elements (referent kinematic elements) attached to the nacelle. This modeling method turns out to be dependent on the mobile platform position and the orientation of referent elements. According to the same generic methodology which is applied to the geometric and kinematic model, a vision-based control was introduced. The image projection of the referent element of the robot was used as feedback signal.

The main advantage of the proposed approaches is that modeling and control are unified according to a coherent representation of parallel robots, in which one can fuse a generic architecture, the kinematic modeling, referent kinematic elements observation and control, for several types of parallel robots. Furthermore, we do not require the use of an additional visual pattern to calibrate the relative pose of the latter with respect to the end-effector.

The proposed generic architecture is based on the characteristics of different parallel robot families and some assumptions, such as an actuated or passive joint, the number of actuators and the number of kinematic elements in each kinematic chain. Despite these assumptions, we proved that a broad class of parallel robots (section II) can be included in this architecture. The modeling and control proved to be well founded in experimental validations on two different families of parallel robots (Gough-Stewart platform and a Par4 robot) presented in this paper.

The proposed generic control is based on the referent kinematic elements observation. Three Cartesian kinematic matrices are computed in generic form: the joint kinematic matrix, the Cartesian kinematic matrix associated to the referent kinematic elements' direction and the Cartesian kinematic matrix associated to the edges (see section $\mathrm{V}$ 
for more details). We do agree that studying the convergence properties of this control algorithm could be very significant. The goal is to find the region of attraction of the controller (global or local), since this is a pure pseudoinverse control law. Authors in ref. [26] studied the controllability of parallel robots using only the visual servoing of leg orientation in Cartesian space. In this case, the Cartesian kinematic matrix associated to the edges shown in Section 5.3, was not considered in the control law. Based on the Hidden robot concept, authors proved that a global diffeomorphism between the Cartesian space and the leg direction space does not always exist. They also certified that the robot did not converge to the local minima, through the application of tools developed for the singularity analysis of robots.

However, research studies in ref. [20] have proven that servo edges might be very accurate than servo orientation for intrinsically geometrical reasons. In this paper, a generic representation of the approach in ref. [20] was proposed. The mapping between the edges and the observed elements' directions (the Cartesian kinematic matrix associated to the edges) is shown in Section 5.3. Hence, part of our future studies will be dedicated to the extension of the approach to other classes of parallel robots and to the study of the controllability of parallel robots using edge-based visual servoing in image space.

\section{References}

1. L. Weiss, A. Sanderson, and C. Neuman, "Dynamic sensor-based control of robots with visual feedback," IEEE Transactions on Robotics and Automation, vol. 3, no. 5, (1987).

2. B. Espiau, F. Chaumette, and P. Rives, "A new approach to visual servoing in robotics," IEEE Transactions on Robotics and Automation, vol. 8, no. 3, pp. 313-326, (1992).

3. S. Hutchinson, G. Hager, and P. Corke, "A tutorial on visual servo control," IEEE Transactions on Robotics and Automation, vol. 12, no. 5, pp. 651-670, (1996).

4. J. Angeles, Fundamentals of Robotic Mechanical Systems: Theory, Methods, and Algorithms. Secaucus, NJ, USA: Springer-Verlag New York, Inc., (1995).

5. J. Merlet, Parallel robots. Kluwer Academic Publishers, (2000).

6. M. Koreichi, S. Babaci, F. Chaumette, G. Fried, and J. Pontnau, "Visual servo control of a parallel manipulator for assembly tasks," 6th International Symposium on Intelligent Robotic Systems, pp. 109116, (1998).

7. P. Kallio, Q. Zhou, and H. N. Koivo, "Three-dimensional position control of a parallel micromanipulator using visual servoing," Microrobotics and Microassembly II, Proceedings of SPIE, vol. 4194, pp. 103-111, (2000).

8. P. Corke, Robotics, Vision and Control. Springer, 2017.

9. M. Husty, "An algorithm for solving the direct kinematics of general Gough-Stewart platforms," Mechanism and Machine Theory, vol. 31, no. 4, pp. 365-380, (1996).

10. J. P. Merlet, "An algorithm for the forward kinematics of general 6 d.o.f parallel manipulators," Research Report 1331, (1990).

11. W. J. Wilson, C. C. Williams Hulls, and G. S. Bell, "Relative end-effector control using cartesian position-based visual servoing," IEEE Transactions on Robotics and Automation, vol. 12, no. 5, pp. 684-696, (1996).

12. B. Thuilot, P. Martinet, L. Cordesses, and J. Gallice, "Position based visual servoing: keeping the object in the field of vision," IEEE ICRA, pp. 1624-1629, (2002).

13. T. Dallej, N. Andreff, Y. Mezouar, and P. Martinet, "3D pose visual servoing relieves parallel robot control from joint sensing," Proceedings of the IEEE International Conference on Intelligent Robots and Systems, pp. 4291-4296, (2006).

14. Z. Qi and J. E. Mcinroy, "Improved image based visual servoing with parallel robot," Journal of Intelligent and Robotic Systems, vol. 53, pp. 359-379, December 2008.

15. G. Palmieri, M. Palpacelli, M. Battistelli, and M. Callegari, "A comparison between position-based and image-based dynamic visual servoings in the control of a translating parallel manipulator," Journal of robotics, vol. 2012, pp. Article ID 103954-, January 2012.

16. F. Chaumette, Potential problems of stability and convergence in image-based and position-based visual servoing. LNCIS Series, No 237, Springer-Verlag, 1998.

17. L. Barron and J. Angeles, "The on-line directs kinematics of parallel manipulators under joint-sensor redundancy," In Advances in Robot Kinematics, pp. 126-137, (1998).

18. L. Tancredi, M. Teillaud, and J.-P. Merlet, "Forward kinematics of a parallel manipulator with additional rotary sensors measuring the position of platform joints," in Computational Kinematics (J.-P. Merlet and B. Ravani, eds.), pp. 261-270, Kluwer Academic Publishers, (1995).

19. N. Andreff, A. Marchadier, and P. Martinet, "Vision-based control of a Gough-Stewart parallel mechanism using legs observation," Proceedings International Conference Robotics and Automation, pp. 2546-2551, (2005). 
20. N. Andreff, T. Dallej, and P. Martinet, "Image-based visual servoing of Gough-Stewart parallel manipulators using legs observation," IJRR International Journal of Robotics Research, vol. 26, no. 7, (2007).

21. T. Dallej, N. Andreff, and P. Martinet, "Visual servoing of par4 using leg observation," IEEE Industrial Electronics Society, pp. 3782-3787, (2006).

22. N. Andreff and P. Martinet, "Kinematic modelling of some parallel manipulators for control purposes," The first European Conference on Mechanism Science, (2006).

23. E. Ozgur, N. Bouton, N. Andreff, and M. Martinet, "Dynamic control of the quattro robot by the leg edges," IEEE International Conference on Robotics and Automation, pp. 2731-2736, (2011).

24. P. Renaud, N. Andreff, P. Martinet, and G. Gogu, "Kinematic calibration of parallel mechanisms: a novel approach using legs observation," IEEE Transactions on Robotics, vol. 21, no. 4, pp. 529-538, (2005).

25. S. Briot and P. Martinet, "Minimal representation for the control of gough-stewart platforms via leg observation considering a hidden robot model," IEEE International Conference on Robotics and Automation, pp. 4653-4658, (2013).

26. S. Briot, V. Rosenzveig, P. Martinet, E. Ozgür, and N. Bouton, "Minimal Representation for the Control of Parallel Robots via Leg Observation Considering a Hidden Robot Model," Mechanism and Machine Theory, vol. 106, (2016).

27. T. Dallej, Contribution to a generic model for visual servoing of parallel robots using legs observation. PhD thesis, Université Blaise Pascal - Clermont-Ferrand II, (2007).

28. E. Ozgür, N. Andreff, and P. Martinet, "Linear dynamic modeling of parallel kinematic manipulators from observable kinematic elements," Mechanism and Machine Theory, vol. 69, pp. 73-89, (2013).

29. F. Pierrot and O. Company, "H4: A new family of 4-dof parallel robots," IEEE International Conference on Advanced Intelligent Mechatronics, pp. 508-513, (1999).

30. S. Krut, V. Nabat, O. Company, and F. Pierrot, "A high-speed parallel robot for scara motions," IEEE International Conference on Robotics and Automation, pp. 4109-4115, (2004).

31. V. Nabat, O. Company, S. Krut, M. Rodriguez, and F. Pierrot, "Par4: Very high speed parallel robot for pick-and-place," IEEE International Conference on Intelligent Robots and Systems, pp. 1202-1207, (2005).

32. S. Krut, O. Company, M. Benoit, H. Ota, and F. Pierrot, "I4: A new parallel mechanism for scara motions," IEEE International Conference on Robotics and Automation, pp. 1875-1880, (2003).

33. F. Gaoa, W. Lia, X. Zhaob, Z. Jinc, and H. Zhaoc, "New kinematic structures for 2-, 3-, 4-, and 5-DOF parallel manipulator designs," Mechanism and machine theory, vol. 37, no. 11, pp. 1395-1411, (2002).

34. P. Wenger and D. Chablat, "Kinematic analysis of a new parallel machine tool: The orthoglide," "th International Symposium on Advances in Robot Kinematics, pp. 305-314, (2000).

35. G. Gogu, Fully-isotropic T3R1-type parallel manipulator. In Advances In Robot Kinematics (eds J. Lenarcic and C. Galletti), Kluwer Academic, (2004).

36. G. Gogu, Structural Synthesis of Parallel Robots Part 5: Basic Overconstrained Topologies with Schönflies Motions. New York, Springer, (2004).

37. C. Gosselin and J. F. Hamel, "The agile eye: A high performance three-degree-of-freedom cameraorienting device," IEEE International Conference on Robotics and Automation, pp. 781-787, (1994).

38. N. Seward and I. Bonev, "A new 6-dof parallel robot with simple kinematic model," IEEE International Conference on Robotics and Automation, (2014).

39. A. Schoenflies, Geometrie der Bewegung in Synthetischer Darstellung. New York, Springer, (1886).

40. J. M. Hervé, "The lie group of rigid body displacements, a fundamental tool for mechanism design," Mechanism and Machine Theory, vol. 34, pp. 719-730, (1999).

41. J. Plücker, "On a new geometry of space," Philosophical Transactions of the Royal Society of London, vol. 155, pp. 725-791, (1865).

42. N. Andreff, B. Espiau, and R. Horaud, "Visual servoing from lines," International Journal of Robotics Research, vol. 21, no. 8, pp. 679-700, (2002).

43. E. Marchand, F. Spindler, and F. Chaumette, "Visp for visual servoing: a generic software platform with a wide class of robot control skills," IEEE Robotics and Automation Magazine, Special Issue on "Software Packages for Vision-Based Control of Motion", P. Oh, D. Burschka (Eds.), vol. 12, no. 4, pp. $40-52,(2005)$.

44. E. Ozgür, N. Andreff, R. Dahmouche, and P. Martinet, "High Speed Parallel Kinematic Manipulator State Estimation from Legs Observation," IEEE/RSJ International Conference on Intelligent Robots and Systems, pp. 426-429, (2013).

45. E. Coronado, M. Maya Mendez, A. Cardenas, O. Guarneros, and D. Piovesan, "Vision-based control of a delta parallel robot via linear camera-space manipulation," Journal of Intelligent and Robotic Systems, 2016.

46. A. Dumlu, K. Erenturk, A. Kaleli, and K. K. Ayten, "A comparative study of two model-based control techniques for the industrial manipulator," Robotica, vol. 35, no. 10, pp. 2036-2055, (2017).

47. J. M. Rossell, J. Vicente-Rodrigo, J. Rubio-Massegu, and V. Barcons, "An effective strategy of real-time vision-based control for a stewart platform," IEEE International Conference on Industrial Technology (ICIT), pp. 75-80, (2018).

48. T. Dallej, N. Andreff, and P. Martinet, "Image-based visual servoing of the I4R parallel robot without proprioceptive sensors," IEEE International Conference on Robotics and Automation, (2007). 
49. T. Dallej, H. Hadj Abdelkader, N. Andreff, and P. Martinet, "Kinematic calibration of a gough-stewart platform using an omnidirectional camera," IEEE International Conference on Intelligent Robots and Systems, pp. 4666-4671, (2006).

50. O. Tahri, Y. Mezouar, N. Andreff, and P. Martinet, "Visual servoing of gough-stewart platform using omnidirectional camera," IEEE Transactions on Robotics and Automation, vol. 25, no. 1, pp. 178-183, (2009). 